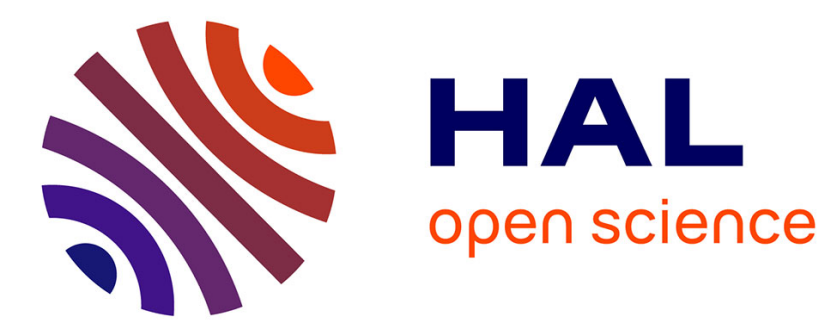

\title{
Local indeterminacy in continuous-time models: the role of returns to scale
}

Jean-Philippe Garnier, Kazuo Nishimura, Alain Venditti

\section{To cite this version:}

Jean-Philippe Garnier, Kazuo Nishimura, Alain Venditti. Local indeterminacy in continuous-time models: the role of returns to scale. 2008. halshs-00281382

\section{HAL Id: halshs-00281382 \\ https://shs.hal.science/halshs-00281382}

Preprint submitted on 22 May 2008

HAL is a multi-disciplinary open access archive for the deposit and dissemination of scientific research documents, whether they are published or not. The documents may come from teaching and research institutions in France or abroad, or from public or private research centers.
L'archive ouverte pluridisciplinaire HAL, est destinée au dépôt et à la diffusion de documents scientifiques de niveau recherche, publiés ou non, émanant des établissements d'enseignement et de recherche français ou étrangers, des laboratoires publics ou privés. 


\section{GREQAM}

Document de Travail

Groupement de Recherche en Economie Quantitative d'Aix-Marseille - UMR-CNRS 6579 Ecole des Hautes Etudes en Sciences Sociales Universités d'Aix-Marseille II et III

LOCAL INDETERMINACY IN CONTINUOUS-TIME MODELS: THE ROLE OF RETURNS TO SCALE

Jean-Philippe GARNIER Kazuo NISHIMURA Alain VENDITTI

May 2008 $n^{\circ} 2008-21$

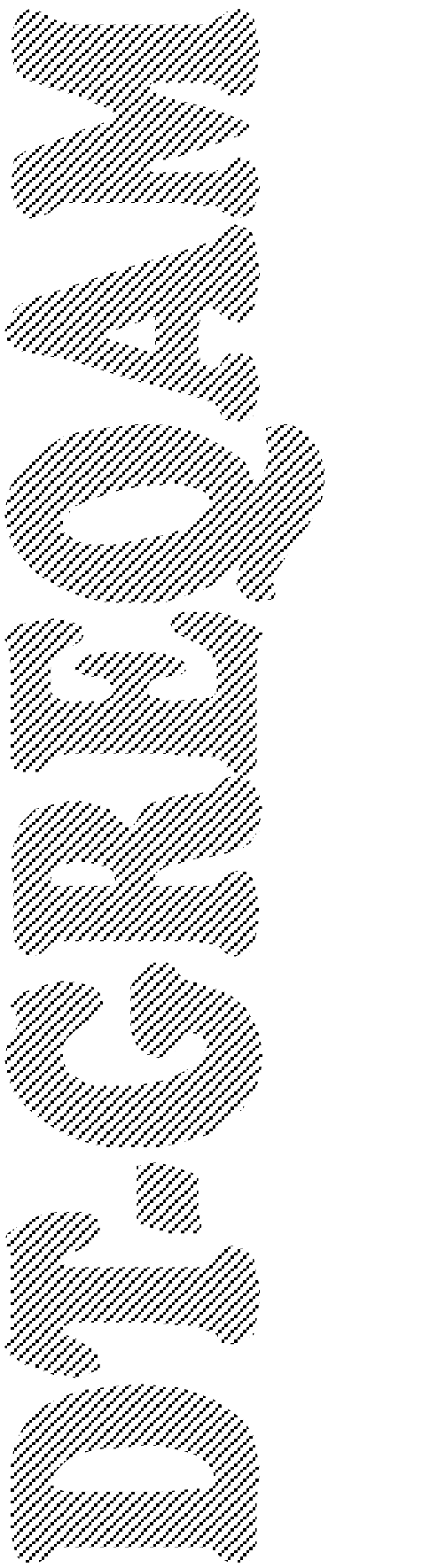




\title{
Local indeterminacy in continuous-time models: the role of returns to scale*
}

\author{
Jean-Philippe GARNIER \\ Université de la Méditerranée, GREQAM, Marseille, France \\ Kazuo NISHIMURA \\ Institute of Economic Research, Kyoto University, Japan \\ and \\ Alain VENDITTI ${ }^{\dagger}$ \\ CNRS - GREQAM, Marseille, France, \\ and \\ Institute of Economic Research, Kyoto University, Japan
}

First version: July 2007; Revised: April 2008

\begin{abstract}
The aim of this paper is to discuss the role of the returns to scale at the private and social levels on the local determinacy properties of the steady state in a continuous-time two-sector economy with sector-specific externalities. First we show that when labor is inelastic, for any configuration of the returns to scale, local indeterminacy is obtained if there is a capital intensity reversal between the private and the social levels. Second, we prove that when labor is infinitely elastic, saddle-point stability is obtained as soon as the investment good sector has constant social returns while local indeterminacy arises if the investment good sector has increasing social returns, provided the consumption good is labor intensive at the social level and the elasticity of intertemporal substitution in consumption admits intermediary values. Finally, we show that local indeterminacy requires a low elasticity of labor when the investment good has constant social returns, but requires either low enough or large enough elasticity of labor when the investment good has increasing social returns.
\end{abstract}

Keywords: Sector-specific externalities, constant and increasing returns, intertemporal substitution in consumption, elastic labor, indeterminacy.

Journal of Economic Literature Classification Numbers: C62, E32, O41.

${ }^{*}$ We are grateful to R. Dos Santos Ferreira and J.M. Grandmont for useful comments and suggestions on a previous paper that have allowed us to initiate this research. This paper also benefited from a presentation at the "Workshop on Mathematical Economics", Keio University, Kyoto, October 2007. We thank T. Maruyama for his kind invitation.

${ }^{\dagger}$ This paper has been written while Alain Venditti was visiting the Institute of Economic Research of Kyoto University. 


\section{Introduction}

The existence of local indeterminacy in infinite-horizon models with externalities is a well established fact. ${ }^{1}$ As initially shown by Benhabib and Farmer [2], this conclusion is generally a consequence of large enough increasing returns at the social level, a large enough elasticity of intertemporal substitution in consumption and a large enough elasticity of the labor supply. $^{2}$ It is worth noticing however that all these contributions deal either with an agregate model or with a two-sector model characterized by identical technologies at the private level.

As increasing returns do not clearly appear to be supported by empirical evidences, ${ }^{3}$ Benhabib and Nishimura [5] have shown in a seminal contribution that local indeterminacy is also compatible with constant returns at the social level in a two-sector model with sector-specific externalities. Assuming a linear utility function with respect to consumption, they have proved that this result is based on a capital intensity reversal, the consumption good being capital intensive at the private level but labor intensive at the social level. More interestingly, this result is established without any restriction on the elasticity of labor supply. ${ }^{4}$

Generalizing the Benhabib-Nishimura formulation by the consideration of a non-linear utility function with respect to consumption, Nishimura and Venditti [16] and Garnier, Nishimura and Venditti [8] have shown that while local indeterminacy is fundamentaly based on a large elasticity of intertemporal substitution in consumption, it requires a low enough elasticity of the labor supply. Moreover, they have proved that saddle-point stability is ensured as soon as the elasticity of the labor supply is large enough. Clearly, this last conclusion appears to be in complete opposition with the results derived under increasing social returns within aggregate or two-sector mod-

\footnotetext{
${ }^{1}$ See Benhabib and Farmer [4] for a survey of the literature.

${ }^{2}$ See also among others Benhabib and Farmer [3], Lloyd-Braga, Nourry and Venditti [6], Nishimura, Nourry and Venditti [14], Pintus [17], Wen [18].

${ }^{3}$ See Basu and Fernald [1].

${ }^{4}$ See also Benhabib, Nishimura and Venditti [6].
} 
els. Our goal in the present paper is to understand such a drastic difference between the two formulations. We will then precisely examine the role of returns to scale on the occurrence of local indeterminacy.

We consider a two-sector model with Cobb-Douglas technologies, sectorspecific externalities, endogenous labor and a non-linear separable CES utility function. Contrary to the Benhabib and Farmer [3] formulation, we consider different technologies at the private level (the capital intensity difference at the private level is non zero) in order to deal with a more general model. Moreover, we assume that one of the two sectors has constant social returns to scale while the other sector is characterized by constant returns either at the private or at the social level. Proceeding that way, we get a framework compatible with both the Benhabib-Farmer [3] and the Benhabib-Nishimura [5] formulations.

We first show that when labor is inelastic, for any of the two configurations for the returns to scale, local indeterminacy is obtained under the same type of condition exhibited by Benhabib and Nishimura [5], namely a capital intensity reversal between the private and the social levels. We then clearly establish the robustness of this condition in particular when the social returns to scale are increasing.

Second, we prove that when labor is infinitely elastic, saddle-point stability is obtained as soon as the investment good sector has constant social returns. On the contrary, if the investment good sector has increasing social returns, local indeterminacy may arise provided the consumption good is labor intensive at the social level and the elasticity of intertemporal substitution in consumption admits intermediary values. Considering these two results simultaneously we finally prove that local indeterminacy requires a low elasticity of labor when the investment good has constant social returns, but requires either low enough or large enough elasticity of labor when the investment good has increasing social returns. More generally, we show that when mild externalities, i.e. weakly increasing social returns in the investment good sector, are considered, local indeterminacy is ruled out for intermediary values of the elasticity of labor supply. To summarize, we show that the drastic difference between the Benhabib-Farmer [3] and the 
Benhabib-Nishimura [5] formulations fundamentaly relies on the returns to scale at the social level in the investment good sector.

The rest of the paper is organized as follows: Section 2 presents the basic model, the intertemporal equilibrium, the steady state and the characteristic polynomial. The main results are exposed in Section 3. Some concluding comments are provided in Section 4 and a final Appendix contains all the proofs.

\section{The model}

\subsection{The production structure}

We consider an economy producing a pure consumption good $y_{0}$ and a pure capital good $y_{1}$. Each good is assumed to be produced by capital $x_{1 j}$ and labor $x_{0 j}, j=0,1$, through a Cobb-Douglas technology which contains sector specific externalities. The representative firm in each industry indeed faces the following technology, called private production function:

$$
y_{j}=x_{0 j}^{\beta_{0 j}} x_{1 j}^{\beta_{1 j}} e_{j}\left(X_{0 j}, X_{1 j}\right), \quad j=0,1
$$

with $\beta_{i j}>0$. The positive externalities are equal to

$$
e_{j}\left(X_{0 j}, X_{1 j}\right)=X_{0 j}^{b_{0 j}} X_{1 j}^{b_{1 j}}
$$

with $b_{i j} \geq 0$ and $X_{i j}$ denoting the average use of input $i$ in sector $j$. We assume that these economy-wide averages are taken as given by each individual firm. At the equilibrium, since all firms of sector $j$ are identical, we have $X_{i j}=x_{i j}$ and we may define the social production functions as follows

$$
y_{j}=x_{0 j}^{\hat{\beta}_{0 j}} x_{1 j}^{\hat{\beta}_{1 j}}
$$

with $\hat{\beta}_{i j}=\beta_{i j}+b_{i j}$. In order to consider mild external effects and to avoid endogenous growth, we will assume that $\hat{\beta}_{i j}<1, i, j=0,1$. We do not a priori impose any restriction on the returns to scale at the private and social levels. However, we will be interested in the analysis of two standard configurations: a first one with constant social returns and thus decreasing private returns, a second one with constant private returns and thus increasing social returns. Total labor is given by $\ell=x_{00}+x_{01}$, and the total stock of capital is given by $x_{1}=x_{10}+x_{11}$. 
Choosing the consumption good as the numeraire, i.e. $p_{0}=1$, a firm in each industry maximizes its profit given the output price $p_{1}$, the rental rate of capital $w_{1}$ and the wage rate $w_{0}$. The first order conditions subject to the private technologies (1) give

$$
a_{i j} \equiv x_{i j} / y_{j}=p_{j} \beta_{i j} / w_{i}, \quad i, j=0,1
$$

We call $a_{i j}$ the input coefficients from the private viewpoint.

Considering that the total labor is given by $\ell=x_{00}+x_{01}$, and the total stock of capital is given by $x_{1}=x_{10}+x_{11}$, the factor market clearing equation is directly obtained from the private input coefficients as defined by (3):

Lemma 1. Denote $x=\left(\ell, x_{1}\right)^{\prime}, y=\left(y_{0}, y_{1}\right)^{\prime}$ and $A=\left[a_{i j}\right] \equiv\left[p_{j} \beta_{i j} / w_{i}\right]$. Then $A y=x$.

Using the fact that the input coefficients form the private viewpoint $a_{i j}$ are functions of $w$ and $p$, i.e. $a_{i j}=p_{j} \beta_{i j} / w_{i}=a_{i j}\left(w_{i}, p_{j}\right)$, a total differenciation of the above factor market clearing equation gives:

Lemma 2. Denote $d x=\left(d \ell, d x_{1}\right)^{\prime}$ and $d y=\left(d y_{0}, d y_{1}\right)^{\prime}$. Then

$$
d x=A d y+\left(\begin{array}{l}
{\left[\frac{\partial a_{00}}{\partial w_{0}} y_{0}+\frac{\partial a_{01}}{\partial w_{0}} y_{1}\right] d w_{0}+\frac{\partial a_{01}}{\partial p_{1}} y_{1} d p_{1}} \\
{\left[\frac{\partial a_{10}}{\partial w_{1}} y_{0}+\frac{\partial a_{11}}{\partial w_{1}} y_{1}\right] d w_{1}+\frac{\partial a_{11}}{\partial p_{1}} y_{1} d p_{1}}
\end{array}\right)
$$

From (3) we get $p_{j}=a_{i j} w_{i} / \beta_{i j}$ or equivalently $p_{j} \hat{\beta}_{i j}=a_{i j} w_{i}\left(\hat{\beta}_{i j} / \beta_{i j}\right)$. Let us then define

$$
\hat{a}_{i j}=\frac{\hat{\beta}_{i j}}{\beta_{i j}} a_{i j}
$$

We get $p_{j} \hat{\beta}_{i j}=\hat{a}_{i j} w_{i}$, and taking the sum over $i$ gives the price equation

$$
p=\left(\begin{array}{cc}
\frac{1}{\hat{\beta}_{00}+\hat{\beta}_{10}} & 0 \\
0 & \frac{1}{\hat{\beta}_{01}+\hat{\beta}_{11}}
\end{array}\right) \hat{A}^{\prime} w
$$

with $p=\left(1, p_{1}\right)^{\prime}, w=\left(w_{0}, w_{1}\right)^{\prime}$ and $\hat{A}=\left[\hat{a}_{i j}\right]$. We will call $\hat{a}_{i j}$ the input coefficients from the social viewpoint. ${ }^{5}$ Substituting the first order conditions

\footnotetext{
${ }^{5}$ If the agents take account of externalities as endogenous variables in profit maximization, the first order conditions subject to the social technologies (2) give these social input coefficients.
} 
(3) into the social production function (2) and solving for $p_{j}$ gives an expression of the input coefficients from the social viewpoint $\hat{a}_{i j}$ as a function of $w$ and $y$ :

$$
\hat{a}_{i j}=\left(\hat{\beta}_{i j} / w_{i}\right) y_{j}^{\frac{1-\hat{\beta}_{0 j}-\hat{\beta}_{1}}{\hat{\beta}_{0 j}+\hat{\beta}_{1 j}}}\left(\hat{\beta}_{0 j} / w_{0}\right)^{\frac{\hat{\beta}_{0 j}}{\hat{\beta}_{0 j}+\hat{\beta}_{1 j}}}\left(\hat{\beta}_{1 j} / w_{1}\right)^{\frac{\hat{\beta}_{0 j}+\hat{\beta}_{1 j}}{\hat{\beta}_{0 j}}}
$$

Substituting this expression into (6) gives the factor-price frontier:

Lemma 3. Denote $p=\left(1, p_{1}\right)^{\prime}$. Then:

$$
p=\left(\begin{array}{cc}
y_{0}^{\frac{1-\hat{\beta}_{00}-\hat{\beta}_{10}}{\hat{\beta}_{00}+\hat{\beta}_{10}}} & 0 \\
0 & y_{1}^{\frac{1-\hat{\beta}_{01}-\hat{\beta}_{11}}{\hat{\beta}_{01}+\hat{\beta}_{11}}}
\end{array}\right)\left(\begin{array}{c}
\left(\frac{w_{0}}{\hat{\beta}_{00}}\right)^{\frac{\hat{\beta}_{00}}{\hat{\beta}_{00}+\hat{\beta}_{10}}}\left(\frac{w_{1}}{\beta_{10}}\right)^{\frac{\hat{\beta}_{10}}{\hat{\beta}_{00}+\hat{\beta}_{10}}} \\
\left(\frac{w_{0}}{\hat{\beta}_{01}}\right)^{\frac{\hat{\beta}_{01}}{\hat{\beta}_{01}+\hat{\beta}_{11}}}\left(\frac{w_{1}}{\beta_{11}}\right)^{\frac{\hat{\beta}_{11}}{\hat{\beta}_{01}+\hat{\beta}_{11}}}
\end{array}\right)
$$

Using the fact that the input coefficients from the social viewpoint $\hat{a}_{i j}$ are functions of $w$ and $y$, a direct differenciation of the factor-price frontier gives:

Lemma 4. Denote $d p=\left(0, d p_{1}\right)^{\prime}$ and $d w=\left(d w_{0}, d w_{1}\right)^{\prime}$. Then

$$
d p=\left(\begin{array}{cc}
\frac{1}{\hat{\beta}_{00}+\hat{\beta}_{10}} & 0 \\
0 & \frac{1}{\hat{\beta}_{01}+\hat{\beta}_{11}}
\end{array}\right) \hat{A}^{\prime} d w+\left(\begin{array}{c}
\frac{1-\hat{\beta}_{00}-\hat{\beta}_{10}}{\hat{\beta}_{00}+\hat{\beta}_{10}} \frac{1}{y_{0}} d y_{0} \\
\frac{1-\hat{\beta}_{01}-\hat{\beta}_{11}}{\hat{\beta}_{01}+\hat{\beta}_{11}} \frac{p}{y} d y_{1}
\end{array}\right)
$$

Notice that the first term on the right-hand-side is obtained from the equivalence between (6) and (7).

Solving equations (4) and (8) with respect to $d y$ and $d w$ allows to compute the partial derivatives $[d y / d x],[d y / d p],[d w / d x]$ and $[d w / d p]$ (see Appendix 5.2 for details). In the following we will then treat $y$ and $w$ as functions of $x$ and $p$, namely $y_{j}=\tilde{y}_{j}\left(x_{1}, \ell, p_{1}\right)$ and $w_{j}=\tilde{w}_{j}\left(x_{1}, \ell, p_{1}\right), j=0,1{ }^{6}$

\subsection{Intertemporal equilibrium and steady state}

The economy is populated by a large number of identical infinitely-lived agents. We assume without loss of generality that the total population

${ }^{6}$ Notice that if the returns to scale are constant at the social level, then $1-\hat{\beta}_{0 j}-\hat{\beta}_{1 j}=0$ and the factor prices $w$ are functions of the output price only, i.e. $w_{j}=w_{j}\left(p_{1}\right), j=0,1$. 
is constant and normalized to one. At each period a representative agent supplies elastically an amount of labor $\ell \in(0, \bar{\ell})$, with $\bar{\ell}=1$ his endowment of labor. He then derives utility from consumption $c$ and leisure $\mathcal{L}=1-\ell$ according to the following function

$$
u(c, \ell)=\frac{c^{1-\sigma}}{1-\sigma}-\frac{\ell^{1+\gamma}}{A(1+\gamma)}
$$

with $\sigma \geq 0, \gamma \geq 0$ and $A$ a normalization constant. The elasticity of intertemporal substitution in consumption is thus given by $\epsilon_{c}=1 / \sigma$ while the elasticity of the labor supply is given by $\epsilon_{\ell}=1 / \gamma$. Considering the external effects $\left(e_{0}, e_{1}\right)$ as given, profit maximization in both sectors described in Section 2.1 gives demands for capital and labor as functions of the capital stock, the production level of the investment good, total labor and the external effects, namely $\tilde{x}_{i j}=x_{i j}\left(x_{1}, y_{1}, \ell, e_{0}, e_{1}\right), i, j=0,1$. The production frontier is then defined as

$$
c=T\left(x_{1}, y_{1}, \ell, e_{0}, e_{1}\right)=\tilde{x}_{00}^{\beta_{00}} \tilde{x}_{10}^{\beta_{10}} e_{0}
$$

From the envelope theorem we easily get $w_{1}=T_{1}\left(x_{1}, y_{1}, \ell, e_{0}, e_{1}\right), p_{1}=$ $-T_{2}\left(x_{1}, y_{1}, \ell, e_{0}, e_{1}\right)$ and $w_{0}=T_{3}\left(x_{1}, y_{1}, \ell, e_{0}, e_{1}\right)$.

The intertemporal optimization problem of the representative agent can be described as:

$$
\begin{array}{cl}
\max _{\left\{x_{1}(t), y_{1}(t), \ell(t)\right\}} & \int_{0}^{+\infty}\left[\frac{T\left(x_{1}(t), y_{1}(t), \ell(t), e_{0}(t), e_{1}(t)\right)^{1-\sigma}}{1-\sigma}-\frac{\ell(t)^{1+\gamma}}{A(1+\gamma)}\right] e^{-\delta t} d t \\
\text { s.t. } & \dot{x}_{1}(t)=y_{1}(t)-g x_{1}(t) \\
& x_{1}(0) \text { given } \\
& \left\{e_{j}(t)\right\}_{t \geq 0}, j=0,1, \text { given }
\end{array}
$$

where $\delta \geq 0$ is the discount rate and $g>0$ is the depreciation rate of the capital stock. We can write the modified Hamiltonian in current value as:

$\mathcal{H}=\frac{T\left(x_{1}(t), y_{1}(t), \ell(t), e_{0}(t), e_{1}(t)\right)^{1-\sigma}}{1-\sigma}-\frac{\ell(t)^{1+\gamma}}{A(1+\gamma)}+q_{1}(t)\left(y_{1}(t)-g x_{1}(t)\right)$ with $q_{1}(t)$ the Lagrange multiplier which corresponds to the utility price of capital in current value. The necessary conditions for a solution of the above dynamic optimization problem are given by the following equations: 


$$
\begin{aligned}
q_{1}(t) & =p_{1}(t) c(t)^{-\sigma} \\
\ell(t)^{\gamma} / A & =w_{0} c(t)^{-\sigma} \\
\dot{x}_{1}(t) & =y_{1}(t)-g x_{1}(t) \\
\dot{q}_{1}(t) & =(\delta+g) q_{1}(t)-w_{1}(t) c(t)^{-\sigma}
\end{aligned}
$$

As shown in Section 2.1, we have in the general case $w_{0}=\tilde{w}_{0}\left(x_{1}, \ell, p_{1}\right)$ and $c=\tilde{y}_{0}\left(x_{1}, \ell, p_{1}\right)=T\left(x_{1}, \tilde{y}_{1}\left(x_{1}, \ell, p_{1}\right), \ell, e_{0}\left(x_{1}, \ell, p_{1}\right), e_{1}\left(x_{1}, \ell, p_{1}\right)\right)$. Therefore, solving equation (10) describing the labor-leisure trade-off at the equilibrium, we may express the labor supply as a function of the capital stock and the output price, $\ell=\ell\left(x_{1}, p_{1}\right)$. Then, we get $y_{0}=$ $c\left(x_{1}, p_{1}\right) \equiv \tilde{y}_{0}\left(x_{1}, \ell\left(x_{1}, p_{1}\right), p_{1}\right), y_{1}=y_{1}\left(x_{1}, p_{1}\right) \equiv \tilde{y}_{1}\left(x_{1}, \ell\left(x_{1}, p_{1}\right), p_{1}\right)$ and $w_{1}=w_{1}\left(x_{1}, p_{1}\right)$. Considering (9)-(12), the equations of motion are finally derived as

$$
\begin{aligned}
& \dot{x}_{1}=y_{1}\left(x_{1}, p_{1}\right)-g x_{1} \\
& \dot{p}_{1}=\frac{1}{E\left(x_{1}, p_{1}\right)}\left[(\delta+g) p_{1}-w_{1}\left(x_{1}, p_{1}\right)+\sigma \frac{p_{1}}{c\left(x_{1}, p_{1}\right)} \frac{\partial c}{\partial x_{1}}\left(y_{1}\left(x_{1}, p_{1}\right)-g x_{1}\right)\right]
\end{aligned}
$$

with

$$
E\left(x_{1}, p_{1}\right)=1-\sigma \frac{p_{1}}{c\left(x_{1}, p_{1}\right)} \frac{\partial c}{\partial p_{1}}
$$

Notice that $E\left(x_{1}, p_{1}\right)$ is equal to 1 minus the product of the inverse of the elasticity of intertemporal substitution in consumption by the elasticity of the consumption good's output with respect to the price of the investment good.

Any solution $\left\{x_{1}(t), p_{1}(t)\right\}_{t \geq 0}$ that also satisfies the transversality condition

$$
\lim _{t \rightarrow+\infty} e^{-\delta t} p_{1}(t) x_{1}(t)=0
$$

is called an equilibrium path.

A steady state is defined by a pair $\left(x_{1}^{*}, p_{1}^{*}\right)$ solution of

$$
\begin{aligned}
y_{1}\left(x_{1}, p_{1}\right) & =g x_{1} \\
w_{1}\left(x_{1}, p_{1}\right) & =(\delta+g) p_{1}
\end{aligned}
$$

In order to simplify the analysis, we use the normalization constant $A$ which enters the utility function in order to choose a particular value for the steady state labor supply. We get the following result: 
Proposition 1. There is a unique value $A^{*}>0$ such that when $A=A^{*}$, there exists a unique steady state $\left(x_{1}^{*}, p_{1}^{*}\right)>0$ with $\ell^{*}=\ell\left(x_{1}^{*}, p_{1}^{*}\right)=\bar{l} \in$ $(0,1)$. Moreover, the expressions $\left(x_{1}^{*}, p_{1}^{*}\right)$ do not depend on the preference parameters $\sigma$ and $\gamma$.

The nice feature of this normalization is that the normalized steady state will remain constant while the elasticity of intertemporal substitution in consumption or the elasticity of the labor supply are varied. This will allow to provide a clear analysis of the local stability properties of the equilibrium path.

\subsection{Characteristic polynomial}

Linearizing the dynamical system (13) around $\left(x_{1}^{*}, p_{1}^{*}\right)$ gives:

$J=\left(\begin{array}{cc}\frac{\partial y_{1}}{\partial x_{1}}-g & \frac{\partial y_{1}}{\partial p_{1}} \\ \frac{1}{E}\left[\sigma \frac{p_{1}^{*}}{c^{*}} \frac{\partial c}{\partial x_{1}}\left(\frac{\partial y_{1}}{\partial x_{1}}-g\right)-\frac{\partial w_{1}}{\partial x_{1}}\right] & \frac{1}{E}\left[\delta+g-\frac{\partial w_{1}}{\partial p_{1}}+\sigma \frac{p_{1}^{*}}{c^{*}} \frac{\partial c}{\partial x_{1}} \frac{\partial y_{1}}{\partial p_{1}}\right]\end{array}\right)$

As we show in Appendix 5.2, all these partial derivatives are functions of $\sigma$ and $\gamma$. The role of $\gamma$ of course occurs through the presence of endogenous labor but remains implicit at that stage mainly because of our methodology to derive the dynamical system (13) from the first order conditions (9)-(12).

Any solution from (13) that converges to the steady state $\left(x_{1}^{*}, p_{1}^{*}\right)$ satisfies the transversality condition and is an equilibrium. Therefore, given $x_{1}(0)$, if there is more than one initial price $p_{1}(0)$ in the stable manifold of $\left(x_{1}^{*}, p_{1}^{*}\right)$, the equilibrium path from $x_{1}(0)$ will not be unique. In particular, if $J$ has two eigenvalues with negative real parts, there will be a continuum of converging paths and thus a continuum of equilibria.

Definition 1. If the locally stable manifold of the steady state $\left(x_{1}^{*}, p_{1}^{*}\right)$ is two-dimensional, then $\left(x_{1}^{*}, p_{1}^{*}\right)$ is said to be locally indeterminate.

The eigenvalues of $J$ are given by the roots of the following characteristic polynomial

$$
\mathcal{P}(\lambda)=\lambda^{2}-\mathcal{T} \lambda+\mathcal{D}
$$

with 


$$
\begin{aligned}
& \mathcal{D}(\sigma, \gamma)=\frac{1}{E}\left\{\left(\frac{\partial y_{1}}{\partial x_{1}}-g\right)\left(\delta+g-\frac{\partial w_{1}}{\partial p_{1}}\right)+\frac{\partial y_{1}}{\partial p_{1}} \frac{\partial w_{1}}{\partial x_{1}}\right\} \\
& \mathcal{T}(\sigma, \gamma)=\frac{1}{E}\left\{\frac{\partial y_{1}}{\partial x_{1}}+\delta-\frac{\partial w_{1}}{\partial p_{1}}+\sigma \frac{p_{1}^{*}}{c^{*}}\left[\frac{\partial c}{\partial x_{1}} \frac{\partial y_{1}}{\partial p_{1}}-\frac{\partial c}{\partial p_{1}}\left(\frac{\partial y_{1}}{\partial x_{1}}-g\right)\right]\right\}
\end{aligned}
$$

(See Appendix 5.2 for the detailed expressions of these derivatives). Local indeterminacy requires therefore that $\mathcal{D}(\sigma, \gamma)>0$ and $\mathcal{T}(\sigma, \gamma)<0$.

Notice from Appendix 5.2 that if constant social returns to scale are assumed in both sectors then $\partial w_{1} / \partial x_{1}=0$ and the Determinant and Trace are equal to the expressions obtained by Benhabib and Nishimura [5] with $\sigma=0$ or by Garnier, Nishimura and Venditti [8] with $\sigma>0$.

\section{Main results}

Our main objective is to study the role of returns to scale at the private and social levels on the local determinacy properties of the long-run equilibrium. In order to simplify the analysis and to consider a framework as close as possible to the formulations of Benhabib and Farmer [3], Harrison [11], Harrison and Weder [12], when one sector will be characterized by increasing social returns, we will assume that the other sector has constant social returns.

We will build the analysis by considering two polar cases. In the first case we assume a linear utility function with respect to labor, i.e. an infinite elasticity of the labor supply $(\gamma=0)$. In the second case we consider the model with inelastic labor $(\gamma=+\infty)$. We will finally derive conclusions for the general case with $\gamma \in(0,+\infty)$.

\subsection{Local indeterminacy with inelastic labor supply}

When the utility function is non-linear with respect to consumption, i.e. $\sigma>0$, and $\gamma=+\infty$, we get a formulation with inelastic labor in which

$$
u(c)=\frac{c^{1-\sigma}}{1-\sigma}
$$

since $\ell \in(0,1)$ and $\lim _{\gamma \rightarrow+\infty} \ell^{1+\gamma} /(1+\gamma)=0$.

As initially shown by Benhabib and Nishimura [5], the local indeterminacy properties within two sector models are based on the capital intensity 
difference across sectors at the private and social levels. Using the definitions of input coefficients given in Section 2.1 allows to characterize these capital intensity differences as follows:

Definition 2. The consumption good is said to be:

i) capital intensive at the private level if and only if $a_{11} a_{00}-a_{10} a_{01}<0$,

ii) capital intensive at the social level if and only if $\hat{a}_{11} \hat{a}_{00}-\hat{a}_{10} \hat{a}_{01}<0$.

We may conveniently relate these input coefficients to the Cobb-Douglas parameters: ${ }^{7}$

Proposition 2. At the steady state:

i) the consumption good is capital (labor) intensive from the private perspective if and only if

$$
b \equiv 1-\frac{\beta_{10} \beta_{01}}{\beta_{00} \beta_{11}}<(>) 0
$$

ii) the consumption good is capital (labor) intensive from the social perspective if and only if

$$
\hat{b} \equiv 1-\frac{\hat{\beta}_{10} \hat{\beta}_{01}}{\hat{\beta}_{00} \hat{\beta}_{11}}<(>) 0
$$

Under constant social returns in both sectors and a linear utility function with respect to consumption $(\sigma=0)$, Benhabib and Nishimura [5] have proved that local indeterminacy arises if the consumption good is capital intensive from the private perspective $(b<0)$, but labor intensive from the social perspective $(\hat{b}>0)$. Building on the same kind of technological restrictions, we obtain the following conclusions:

Theorem 1. Let $\gamma=+\infty$ and consider the critical value

$$
\hat{\sigma}=-\frac{b\left(\hat{\beta}_{00} \hat{\beta}_{11}-\hat{\beta}_{10} \hat{\beta}_{01}\right)\left[\delta+g\left(1-\beta_{11}\right)\right]}{\hat{\beta}_{00}(1-b)(\delta+g)+\hat{\beta}_{10}\left[\delta+g\left(1-\beta_{11} b\right)\right]}
$$

Then the following results hold:

i) When both sectors are characterized by constant social returns, i.e. $1-\hat{\beta}_{00}-\hat{\beta}_{10}=1-\hat{\beta}_{01}-\hat{\beta}_{11}=0$, the steady state is locally indeterminate if and only if

${ }^{7}$ See Benhabib and Nishimura [5]. 


$$
b<0, \hat{b}>0 \text { and } \sigma \in\left[0, \sigma^{*}\right)
$$

with $\sigma^{*}=\hat{\sigma}$.

ii) When the investment good sector is characterized by constant social returns, i.e. $1-\hat{\beta}_{01}-\hat{\beta}_{11}=0$, while the consumption good sector is characterized by increasing social returns, i.e. $1-\hat{\beta}_{00}-\hat{\beta}_{10}<0$, there exists $\bar{g}>0$ such that the steady state is locally indeterminate if

$$
b<0, \quad \hat{b}>-\frac{\left(1-\hat{\beta}_{00}-\hat{\beta}_{10}\right)\left[\delta+g\left(1-\beta_{11} b\right)\right]}{\hat{\beta}_{00}\left[\delta+g\left(1-\beta_{11}\right)\right]}, \quad g \in(0, \bar{g}) \quad \text { and } \quad \sigma \in\left[0, \sigma^{*}\right)
$$

with

$$
\sigma^{*}=\hat{\sigma}-\frac{\left(1-\hat{\beta}_{00}-\hat{\beta}_{10}\right)\left[\hat{\beta}_{01}(1-b)(\delta+g)+\hat{\beta}_{11}\left[\delta+g\left(1-\beta_{11} b\right)\right]\right]}{\hat{\beta}_{00}(1-b)(\delta+g)+\hat{\beta}_{10}\left[\delta+g\left(1-\beta_{11} b\right)\right]}
$$

iii) When the investment good sector is characterized by increasing social returns, i.e. $1-\hat{\beta}_{01}-\hat{\beta}_{11}<0$, while the consumption good sector is characterized by constant social returns, i.e. $1-\hat{\beta}_{00}-\hat{\beta}_{10}=0$, the steady state is locally indeterminate if

$$
b<\frac{\left(1-\hat{\beta}_{01}-\hat{\beta}_{11}\right)(\delta+g)}{\left(1-\hat{\beta}_{11}\right) g}, \quad \hat{b}>0 \quad \text { and } \quad \sigma \in\left[0, \sigma^{*}\right)
$$

with

$$
\sigma^{*}=\hat{\sigma}-\frac{\delta+g\left(1-\beta_{11}\right)}{g} \frac{\left(1-\hat{\beta}_{01}-\hat{\beta}_{11}\right)\left[\delta+g\left(1-\hat{\beta}_{10} \beta_{11} b\right)\right]}{\left[\hat{\beta}_{00}(1-b)(\delta+g)+\hat{\beta}_{10}\left[\delta+g\left(1-\beta_{11} b\right)\right]\right] \beta_{11}}
$$

Notice that all the three configurations require the same restriction on the elasticity of intertemporal substitution in consumption, namely $\varepsilon_{c}=1 / \sigma$ needs to be larger than the inverse of the elasticity of the consumption good's output with respect to the price of the investment good, i.e. $\varepsilon_{c}>1 / \sigma^{*}=$ $\left(p_{1}^{*} / c^{*}\right)\left(\partial c / \partial p_{1}\right)$. This restriction implies thus that $E\left(x_{1}^{*}, p_{1}^{*}\right)>0$. However, increasing returns at the social level allow to get local indeterminacy with lower values for the elasticity of intertemporal substitution in consumption than in the case with constant social returns. As the consumption good is assumed to be capital intensive at the private level, i.e. $b<0$, we have indeed in ii) and iii), $\sigma^{*}>\hat{\sigma}$.

Theorem 1 provides for the first time in the literature a general analysis of local indeterminacy in a real two-sector model with increasing social returns. Indeed, all the previous contributions dealing with increasing social returns were derived from Benhabib and Farmer [3] in which the technologies of both 
sectors are identical at the private level, i.e. in our notations $b=0 .{ }^{8}$ Within such a framework, the technological conditions for local indeterminacy are not related to capital intensity differences across sectors. It follows that comparisons with contributions dealing with constant returns to scale at the social level such as Benhabib and Nishimura [5] or Garnier, Nishimura and Venditti [8] were difficult to obtain. Theorem 1 then shows that the social returns to scale being constant or increasing, local indeterminacy is fundamentaly obtained if there is a capital intensity reversal between the private and the social levels and the elasticity of intertemporal substitution in consumption is large enough.

\subsection{Local indeterminacy with infinitely elastic labor supply}

When the utility function is non-linear with respect to consumption and the labor supply is infinitely elastic, i.e. $\sigma>0$ and $\gamma=0$, we get

$$
u(c, \ell)=\frac{c^{1-\sigma}}{1-\sigma}-\ell
$$

As shown in Benhabib and Farmer [2], Lloyd-Braga, Nourry and Venditti [13], Nishimura, Nourry and Venditti [14] or Pintus [17], the occurrence of local indeterminacy in aggregate models requires the consideration of a large enough (close to infinite) elasticity of the labor supply. Such a restriction is also necessary in two-sector models derived from Benhabib and Farmer [3] in which the technologies of both sectors are identical at the private level $(b=0)$ and the returns to scale are increasing at the social level. However, as we have shown with Theorem 1, two-sector models provide a framework in which local indeterminacy arises through different channels than aggregate models where the intertemporal allocations of labor play a crucial role. Indeed, in a multi-sector framework, the existence of a continuum of equilibria is based on intertemporal reallocations of productive factors between sectors and is fully compatible with inelastic labor, the returns to scale being constant or increasing at the social level.

Of course, an infinite number of equilibria may also occur within a twosector model with elastic labor. The following Theorem then provides an

${ }^{8}$ See Harrison [11], Harrison and Weder [12]. 
analysis of local indeterminacy with an infinitely elastic labor supply. We show that the results fundamentaly depend on the properties of the sectoral returns to scale at the social level.

Theorem 2. Let $\gamma=0$. Then the following results hold:

i) When the investment good sector is characterized by constant social returns, i.e. $1-\hat{\beta}_{01}-\hat{\beta}_{11}=0$, the steady state is saddle-point stable for any $\sigma \geq 0$.

ii) When the investment good sector is characterized by increasing social returns, i.e. $1-\hat{\beta}_{01}-\hat{\beta}_{11}<0$, while the consumption good sector is characterized by constant social returns, i.e. $1-\hat{\beta}_{00}-\hat{\beta}_{10}=0$, there exist $\epsilon>0$, $\underline{\sigma}>0$ and $\bar{\sigma}>\underline{\sigma}$ such that the steady state is locally indeterminate if

$$
\hat{\beta}_{01}+\hat{\beta}_{11} \in(1,1+\epsilon), \quad \hat{b} \in\left(-\frac{\left(1-\hat{\beta}_{01}-\hat{\beta}_{11}\right)(\delta+g)}{\hat{\beta}_{11} \beta_{11} g}, \frac{\hat{\beta}_{01} \beta_{11} \delta}{\hat{\beta}_{00} \hat{\beta}_{11}(\delta+g)}\right) \text { and } \sigma \in(\underline{\sigma}, \bar{\sigma})
$$

In case i), saddle-point stability is obtained for any type of social returns to scale in the consumption good sector, i.e. either when $1-\hat{\beta}_{00}-\hat{\beta}_{10}=0$ or when $1-\hat{\beta}_{00}-\hat{\beta}_{10}<0$. As shown in Appendix 5.4, it is also worth noticing that the conditions for local indeterminacy in case ii) imply that the elasticity of intertemporal substitution in consumption is lower than the elasticty of the consumption good's output with respect to the price of the investment good, i.e. $E\left(x_{1}^{*}, p_{1}^{*}\right)<0$. Actually, we can show that when small externalities are considered, local indeterminacy is ruled out as soon as $E\left(x_{1}^{*}, p_{1}^{*}\right)>0$.

Proposition 3. Let $\gamma=0, \hat{b}>0$ and consider $E\left(x_{1}, p_{1}\right)$ as defined by (14). There exists $\epsilon>0$ such that if $\hat{\beta}_{01}+\hat{\beta}_{11} \in(1,1+\epsilon)$ and $\sigma$ is fixed so as to imply $E\left(x_{1}^{*}, p_{1}^{*}\right)>0$, then $\mathcal{D}(\sigma, 0)<0$ and local indeterminacy is ruled out.

Theorem 2 shows that as soon as the investment good sector is characterized by constant social returns, the steady state is necessarily saddle-point stable for any value of the elasticity of intertemporal substitution in consumption and any amount of external effects. This result explains why local indeterminacy is ruled out in two-sector models with constant social returns 
to scale in both sectors and large values for the elasticity of the labor supply. ${ }^{9}$

On the contrary, when the investment good sector is characterized by slightly increasing social returns and is capital intensive at the social level, local indeterminacy arises for intermediary values of the social capital intensity difference and the elasticity of intertemporal substitution in consumption. Notice that there is no particular restriction on the capital intensity difference at the private level. This fact suggests that our conclusions are compatible with two-sector models in which the private technologies are identical across sectors, i.e. $b=0$, as in Benhabib and Farmer [3], Harrison [11], Harrison and Weder [12]. Notice also that our conclusions hold with almost constant social returns to scale and thus extremely small externalities in the investment good sector.

\subsection{Local indeterminacy with elastic labor supply}

Building on Theorems 1 and 2, we may now provide a complete picture of the existence of local indeterminacy depending on the properties of the social returns to scale in both sectors.

Theorem 3. Let $\gamma \in[0,+\infty)$. The following results hold:

i) When both sectors are characterized by constant social returns, i.e. $1-\hat{\beta}_{00}-\hat{\beta}_{10}=1-\hat{\beta}_{01}-\hat{\beta}_{11}=0$, consider $\sigma^{*}=\hat{\sigma}$ as defined by (19) in Theorem 1. Then for any $\sigma \in\left[0, \sigma^{*}\right)$, there exists a bound $\underline{\gamma}(\sigma)>0$ such that the steady state is locally indeterminate if and only if

$$
b<0, \quad \hat{b}>0
$$

and $\gamma>\underline{\gamma}(\sigma)$.

ii) When the investment good sector is characterized by constant social returns, i.e. $1-\hat{\beta}_{01}-\hat{\beta}_{11}=0$, while the consumption good sector is characterized by increasing social returns, i.e. $1-\hat{\beta}_{00}-\hat{\beta}_{10}<0$, consider $\sigma^{*}$ as defined by (20) and the critical value $\bar{g}$ as given in Theorem 1ii). For any $g \in(0, \bar{g})$ and $\sigma \in\left[0, \sigma^{*}\right)$, there exists a bound $\underline{\gamma}(\sigma)>0$ such that the steady state is locally indeterminate if

\footnotetext{
${ }^{9}$ See Nishimura and Venditti [16] for discrete-time models and Garnier, Nishimura and Venditti [8] for continuous-time models.
} 
and $\gamma>\underline{\gamma}(\sigma)$.

$$
b<0, \quad \hat{b}>-\frac{\left(1-\hat{\beta}_{00}-\hat{\beta}_{10}\right)\left[\delta+g\left(1-\beta_{2} b\right)\right]}{\hat{\beta}_{00}\left[\delta+g\left(1-\beta_{2}\right)\right]}
$$

iii) When the investment good sector is characterized by increasing social returns, i.e. $1-\hat{\beta}_{01}-\hat{\beta}_{11}<0$, while the consumption good sector is characterized by constant social returns, i.e. $1-\hat{\beta}_{00}-\hat{\beta}_{10}=0$, consider $\underline{\sigma}$ and $\bar{\sigma}$ as given in Theorem 2iii). There exist $\epsilon>0, \underline{\gamma}(\sigma)>0$ and $\bar{\gamma}(\sigma)>\underline{\gamma}(\sigma)$ such that for any $\hat{\beta}_{01}+\hat{\beta}_{11} \in(1,1+\epsilon)$ and $\sigma \in(\underline{\sigma}, \bar{\sigma})$, the steady state is locally indeterminate if

$$
b<\frac{\left(1-\hat{\beta}_{01}-\hat{\beta}_{11}\right)(\delta+g)}{\left(1-\hat{\beta}_{11}\right) g}, \hat{b} \in\left(-\frac{\left(1-\hat{\beta}_{01}-\hat{\beta}_{11}\right)(\delta+g)}{\hat{\beta}_{11} \beta_{11} g}, \frac{\hat{\beta}_{01} \beta_{11} \delta}{\hat{\beta}_{00} \hat{\beta}_{11}(\delta+g)}\right)
$$

and $\gamma \in[0, \underline{\gamma}(\sigma)) \cup(\bar{\gamma}(\sigma),+\infty)$.

Theorem 3 shows that as soon as the investment good sector is characterized by constant social returns, the existence of local indeterminacy is based on a capital intensity reversal between the private and the social levels, a large enough elasticity of intertemporal substitution in consumption and a low enough elasticity of the labor supply. On the contrary, when the investment good sector is characterized by slightly increasing social returns, local indeterminacy requires intermediary values for the elasticity of intertemporal substitution in consumption but extreme values for the elasticity of the labor supply. In other words, local indeterminacy is ruled out for intermediary values of $\gamma$. This result explains why all the literature with increasing social returns only focusses on configurations with $\gamma$ close enough to zero. The occurrence of saddle-point stability as $\gamma$ is increased has suggested that local indeterminacy would require a large elasticity of the labor supply as in aggregate models. However, we prove that this is not true as indeterminacy also arises when the labor supply is weakly elastic. Notice also that the consideration of a low elasticity of labor supply appears to be much more empirically plausible as shown by Blundell and McCurdy [7].

Remark: In case i) of Theorems 1 and 3 we provide necessary and sufficient conditions for local indeterminacy as we are able to apply a geometrical methodology initially introduced by Grandmont, Pintus and de Vilder [10]. ${ }^{10}$

${ }^{10}$ See Garnier, Nishimura and Venditti [9] for details. 
Indeed, under constant social returns in both sectors, the determinant $\mathcal{D}$ can be obtained as a linear function of the trace $\mathcal{T}$ when $\sigma$ or $\gamma$ is varied. On the contrary, as soon as increasing social returns to scale are introduced in one of the two sectors, the complexity of the formulation prevents from having a simple relationship between $\mathcal{D}$ and $\mathcal{T}$. That is why we provide only sufficient conditions.

\section{Concluding comments}

We have considered a two-sector economy with CES technologies containing sector-specific externalities and additively separable CES preferences defined over consumption and leisure. We have discussed the existence of local indeterminacy depending on the returns to scale at the private and social level. Two basic configurations have been explored: constant social and decreasing private returns to scale as in Benhabib and Nishimura [5], or increasing social and constant private returns to scale as in Benhabib and Farmer $[2,3]$.

First we have shown that when labor is inelastic, for any configuration of the returns to scale, local indeterminacy is obtained if there is a capital intensity reversal between the private and the social levels. We have then proved the robustness of this condition in particular when the social returns to scale are increasing.

Second, we have shown that when labor is infinitely elastic, saddle-point stability is obtained as soon as the investment good sector has constant social returns. On the contrary, if the investment good sector has increasing social returns, local indeterminacy may arise provided the consumption good is capital intensive at the social level and the elasticity of intertemporal substitution in consumption admits intermediary values.

Finally, we have proved that local indeterminacy requires a low elasticity of labor when the investment good has constant social returns, but requires either low enough or large enough elasticity of labor when the investment good has increasing social returns. This last result clearly shows that the conditions for the existence of local indeterminacy are strongly affected by 
the precise assumptions made on the returns to scale in the investment good sector.

\section{$5 \quad$ Appendix}

\subsection{Proof of Proposition 1}

Maximizing the profit subject to the private technologies (1) gives the first order conditions

$$
p_{j} \beta_{i j} y_{j} / x_{i j}=w_{i}, \quad i, j=0,1
$$

Considering the steady state with $y_{1}=g x_{1}$ and $w_{1}=(\delta+g) p_{1}$, we get

$$
x_{11}=\frac{\beta_{11}}{\delta+g} g x_{1}
$$

Using the social production function (2) for the investment good we derive

$$
x_{01}=\left(\frac{\beta_{11}}{\delta+g}\right)^{-\frac{\hat{\beta}_{11}}{\hat{\beta}_{01}}}\left(g x_{1}\right)^{\frac{1-\hat{\beta}_{11}}{\hat{\beta}_{01}}}
$$

and thus

$$
\frac{x_{01}}{x_{11}}=\left(\frac{\beta_{11}}{\delta+g}\right)^{-\frac{\hat{\beta}_{01}+\hat{\beta}_{11}}{\hat{\beta}_{01}}}\left(g x_{1}\right)^{\frac{1-\hat{\beta}_{01}-\hat{\beta}_{11}}{\hat{\beta}_{01}}}
$$

From $x_{1}=x_{10}+x_{11}$ we derive

$$
x_{10}=x_{1}\left(1-\frac{\beta_{11}}{\delta+g} g\right)
$$

Finally we easily obtain from (22):

$$
\frac{\beta_{10} \beta_{01}}{\beta_{00} \beta_{11}}=\frac{x_{01}}{x_{11}} \frac{x_{10}}{x_{00}} \Leftrightarrow \frac{x_{10}}{x_{00}}=\frac{\beta_{10} \beta_{01}}{\beta_{00} \beta_{11}}\left(\frac{\beta_{11}}{\delta+g}\right)^{\frac{\hat{\beta}_{01}+\hat{\beta}_{11}}{\hat{\beta}_{01}}}\left(g x_{1}\right)^{\frac{\hat{\beta}_{01}+\hat{\beta}_{11}-1}{\hat{\beta}_{01}}}
$$

so that, using (26):

$$
x_{00}=\left(1-\frac{\beta_{11}}{\delta+g} g\right) \frac{\beta_{00} \beta_{11}}{\beta_{10} \beta_{01}}\left(\frac{\beta_{11}}{\delta+g}\right)^{-\frac{\hat{\beta}_{01}+\hat{\beta}_{11}}{\hat{\beta}_{01}}} g^{\frac{1-\hat{\beta}_{01}-\hat{\beta}_{11}}{\hat{\beta}_{01}}} x_{1}^{\frac{1-\hat{\beta}_{11}}{\hat{\beta}_{01}}}
$$

Considering (25), (27) and $x_{00}+x_{01}=\ell, x_{1}=x_{10}+x_{11}$, we get

$$
x_{1}^{*}=\frac{\left(\ell^{*} \frac{\beta_{10} \beta_{01}}{\beta_{00} \beta_{11}}\right)^{\frac{\hat{\beta}_{01}}{1-\hat{\beta}_{11}}}\left(\frac{\beta_{11}}{\delta+g}\right)^{\frac{\hat{\beta}_{01}+\hat{\beta}_{11}}{1-\hat{\beta}_{11}}}}{g^{\frac{1-\hat{\beta}_{01}-\hat{\beta}_{11}}{1-\hat{\beta}_{11}}}\left(1-\frac{\beta_{11}}{\delta+g} g\right)^{\frac{\hat{\beta}_{01}}{1-\hat{\beta}_{11}}}} \equiv\left(\ell^{*}\right)^{\frac{\hat{\beta}_{01}}{1-\hat{\beta}_{11}}} \kappa^{*}
$$

with $b$ given in Proposition 2. Equation (22) for $i=1$ and $j=0$ gives

$$
w_{1}=\beta_{10} x_{00}^{\hat{\beta}_{00}} x_{10}^{\hat{\beta}_{10}-1}
$$


Considering (27), (29) and the fact that $w_{1}=(\delta+g) p_{1}$ implies

$$
\begin{aligned}
p_{1}^{*} & =\frac{\beta_{10}}{\delta+g}\left(1-\frac{\beta_{11}}{\delta+g} g\right)^{\hat{\beta}_{00}+\hat{\beta}_{10}-1}\left(\frac{\beta_{00} \beta_{11}}{\beta_{10} \beta_{01}}\right)^{\hat{\beta}_{00}}\left(\frac{\beta_{11}}{\delta+g}\right)^{-\frac{\hat{\beta}_{00}\left(\hat{\beta}_{01}+\hat{\beta}_{11}\right)}{\hat{\beta}_{01}}} g \frac{\hat{\beta}_{00}\left(1-\hat{\beta}_{01}-\hat{\beta}_{11}\right)}{\hat{\beta}_{01}} \\
& \times\left(\kappa^{*}\right)^{\frac{\hat{\beta}_{00}\left(1-\hat{\beta}_{11}-\hat{\beta}_{01}\left(1-\hat{\beta}_{10}\right)\right.}{\hat{\beta}_{01}}}\left(\ell^{*}\right)^{\frac{\hat{\beta}_{00}\left(1-\hat{\beta}_{11}\right)-\hat{\beta}_{01}\left(1-\hat{\beta}_{10}\right)}{1-\hat{\beta}_{11}}}
\end{aligned}
$$

The substitution of (26) and (28) into (2) gives the expression of $c^{*}$, namely

$$
\begin{aligned}
c^{*} & =\left(1-\frac{\beta_{11}}{\delta+g} g\right)^{\hat{\beta}_{00}+\hat{\beta}_{10}}\left(\frac{\beta_{00} \beta_{11}}{\beta_{10} \beta_{01}}\right)^{\hat{\beta}_{00}}\left(\frac{\beta_{11}}{\delta+g}\right)^{-\frac{\hat{\beta}_{00}\left(\hat{\beta}_{01}+\hat{\beta}_{11}\right)}{\hat{\beta}_{01}}} g^{\frac{\hat{\beta}_{00}\left(1-\hat{\beta}_{01}-\hat{\beta}_{11}\right)}{\hat{\beta}_{01}}} \\
& \times\left(\kappa^{*}\right)^{\frac{\hat{\beta}_{00}\left(1-\hat{\beta}_{11}\right)+\hat{\beta}_{01} \hat{\beta}_{10}}{\hat{\beta}_{01}}}\left(\ell^{*}\right)^{\frac{\hat{\beta}_{00}\left(1-\hat{\beta}_{11}\right)+\hat{\beta}_{01} \hat{\beta}_{10}}{1-\hat{\beta}_{11}}} \\
& \equiv \chi^{*}\left(\ell^{*}\right)^{\frac{\hat{\beta}_{00}\left(1-\hat{\beta}_{11}\right)+\hat{\beta}_{01} \hat{\beta}_{10}}{1-\hat{\beta}_{11}}}
\end{aligned}
$$

Finally, using (22), (28) and (29) into (10) gives

$$
\frac{\left(\ell^{*}\right)^{1+\gamma-\frac{(1-\sigma) \hat{\beta}_{00}\left(1-\hat{\beta}_{11} b\right)}{1-\hat{\beta}_{11}}}}{A}=\frac{\hat{\beta}_{00}\left(\chi^{*}\right)^{1-\sigma}}{1-\frac{\beta_{11}}{\delta+g} g} \frac{\beta_{10} \beta_{01}}{\beta_{00} \beta_{11}}\left(\frac{\beta_{11}}{\delta+g}\right)^{\frac{\hat{\beta}_{01}+\hat{\beta}_{11}}{\hat{\beta}_{01}}} g^{\frac{\hat{\beta}_{01}+\hat{\beta}_{11}-1}{\hat{\beta}_{01}}}\left(\kappa^{*}\right)^{\frac{\hat{\beta}_{11}-1}{\hat{\beta}_{01}}}
$$

As $\ell \in(0,1)$, let us consider a value $\bar{l} \in(0,1)$. It follows therefore that if $A=A^{*}$ with

$A^{*}=\frac{1-\frac{\beta_{11} g}{\delta+g}}{\hat{\beta}_{00}\left(\chi^{*}\right)^{1-\sigma}} \frac{\beta_{00} \beta_{11}}{\beta_{10} \beta_{01}}\left(\frac{\beta_{11}}{\delta+g}\right)^{-\frac{\hat{\beta}_{01}+\hat{\beta}_{11}}{\hat{\beta}_{01}}} g^{\frac{1-\hat{\beta}_{01}-\hat{\beta}_{11}}{\hat{\beta}_{01}}}\left(\kappa^{*}\right)^{\frac{1-\hat{\beta}_{11}}{\hat{\beta}_{01}}}(\bar{l})^{1+\gamma-\frac{(1-\sigma) \hat{\beta}_{00}\left(1-\hat{\beta}_{11} b\right)}{1-\hat{\beta}_{11}}}$ then $\ell^{*}=\bar{l}$ and the result follows.

\subsection{Computation of $\mathcal{D}(\sigma, \gamma)$ and $\mathcal{T}(\sigma, \gamma)$}

Consider the expressions (18) of the Trace and Determinant, and let $y_{0}=$ $c$. We need therefore to compute the following derivatives of $y$ and $w$, namely: $\partial c / \partial x_{1}, \partial c / \partial p_{1}, \partial y_{1} / \partial x_{1}, \partial y_{1} / \partial p_{1}, \partial w_{0} / \partial x_{1}, \partial w_{0} / \partial p_{1}, \partial w_{1} / \partial x_{1}$ and $\partial w_{1} / \partial p_{1}$. We use a procedure by substitution starting from the total differenciations of the factor-price frontier and the factor market clearing equation given in Lemmas 2 and 4 . Solving equation (8) in Lemma 4 with $d x_{1}=0$, we get the derivatives of the wage rate and of the rental rate with respect to the price of investment good:

$$
\begin{aligned}
\frac{d w_{0}}{d p_{1}} & =-\frac{\hat{a}_{10}\left[\hat{\beta}_{01}+\hat{\beta}_{11}-\left(1-\hat{\beta}_{01}-\hat{\beta}_{11}\right) \frac{p_{1}}{y_{1}} \frac{d y_{1}}{d p_{1}}+\left(1-\hat{\beta}_{00}-\hat{\beta}_{10}\right) \frac{\hat{\beta}_{11}}{\hat{\beta}_{10}} \frac{p_{1}}{c} \frac{d c}{d p_{1}}\right]}{\hat{a}_{11} \hat{a}_{00}-\hat{a}_{10} \hat{a}_{01}} \\
\frac{d w_{1}}{d p_{1}} & =\frac{\hat{a}_{00}\left[\hat{\beta}_{01}+\hat{\beta}_{11}-\left(1-\hat{\beta}_{01}-\hat{\beta}_{11}\right) \frac{p_{1}}{y_{1}} \frac{d y_{1}}{d p_{1}}+\left(1-\hat{\beta}_{00}-\hat{\beta}_{10}\right) \frac{\hat{\beta}_{01}}{\hat{\beta}_{00}} \frac{p_{1}}{c} \frac{d c}{d p_{1}}\right]}{\hat{a}_{11} \hat{a}_{00}-\hat{a}_{10} \hat{a}_{01}}
\end{aligned}
$$


Similarly, solving equation (8) in Lemma 4 with $d p_{1}=0$, we get the derivatives of the wage rate and the rental rate with respect to the stock of capital

$$
\begin{aligned}
\frac{d w_{0}}{d x_{1}} & =-\frac{\hat{a}_{01}\left[\left(1-\hat{\beta}_{00}-\hat{\beta}_{10}\right) \frac{\hat{\beta}_{11}}{\hat{\beta}_{10}} \frac{p_{1}}{c} \frac{d c}{d x}-\left(1-\hat{\beta}_{01}-\hat{\beta}_{11}\right) \frac{p_{1}}{y_{1}} \frac{d y_{1}}{d x_{1}}\right]}{\hat{a}_{11} \hat{a}_{00}-\hat{a}_{10} \hat{a}_{01}} \\
\frac{d w_{1}}{d x_{1}} & =\frac{\hat{a}_{00}\left[\left(1-\hat{\beta}_{00}-\hat{\beta}_{10}\right) \frac{\hat{\beta}_{01}}{\hat{\beta}_{00}} \frac{p_{1}}{c} \frac{d c}{d x_{1}}-\left(1-\hat{\beta}_{01}-\hat{\beta}_{11}\right) \frac{p_{1}}{y_{1}} \frac{d y_{1}}{d x_{1}}\right]}{\hat{a}_{11} \hat{a}_{00}-\hat{a}_{10} \hat{a}_{01}}
\end{aligned}
$$

Notice that if we assume constant social returns as in Benhabib and Nishimura [5], then $1-\hat{\beta}_{00}-\hat{\beta}_{10}=1-\hat{\beta}_{00}-\hat{\beta}_{10}=0$. It follows that the rental rate only depends on the price of the investment good as $d w_{1} / d x_{1}=d w_{0} / d x_{1}=0$. Moreover, we derive from (31) that the derivatives $d w_{1} / d p_{1}$ and $d w_{0} / d p_{1}$ depend on the capital intensity difference across sectors at the social level and correspond to the Stolper-Samuelson effects.

Consider now equation (4) in Lemma 2. We easily derive from the definition of input coefficients at the private level (3) that

$$
\frac{\partial a_{0 j}}{\partial w_{0}}=-\frac{a_{0 j}}{w_{0}}, \quad \frac{\partial a_{1 j}}{\partial w_{1}}=-\frac{a_{1 j}}{w_{1}}, \quad \frac{\partial a_{i 1}}{\partial p_{1}}=\frac{a_{i 1}}{p_{1}}
$$

Moreover, we derive from the factor market clearing equation given in Lemma 1 that

$$
\begin{aligned}
& w_{1}=\frac{\hat{a}_{00}}{\hat{a}_{11} \hat{a}_{00}-\hat{a}_{10} \hat{a}_{01}} \frac{p}{\hat{\beta}_{00}}\left[\hat{\beta}_{11} \hat{\beta}_{00}-\hat{\beta}_{10} \hat{\beta}_{01}\right] \\
& w_{0}=\frac{\hat{a}_{10}}{\hat{a}_{11} \hat{a}_{00}-\hat{a}_{10} \hat{a}_{01}} \frac{p}{\hat{\beta}_{10}}\left[\hat{\beta}_{11} \hat{\beta}_{00}-\hat{\beta}_{10} \hat{\beta}_{11}\right]
\end{aligned}
$$

Substituting these expressions into (4) and solving for the derivatives of the two ouput levels with respect to the price of investment good under the restriction $d x_{1}=0$, we get after tedious but straightforward algebra:

$$
\begin{aligned}
\frac{d c}{d p_{1}} & =-\frac{T}{D}\left\{\frac{\hat{T}}{w_{0} w_{1}}\left(\hat{\beta}_{00} a_{01} x_{1}+\hat{\beta}_{10} a_{11} \ell\right)\right. \\
& \left.-\frac{d \ell}{d p_{1}}\left[a_{11}+\hat{T} \hat{a}_{00} \frac{p_{1} x_{1}}{w_{1} y_{1}}\left(1-\hat{\beta}_{01}-\hat{\beta}_{11}\right)\right]\right\} \\
\frac{d y_{1}}{d p_{1}} & =\frac{T}{D}\left\{\frac{\hat{T}}{w_{0} w_{1} c}\left[\left(\hat{\beta}_{00} a_{00} x_{1}+\hat{\beta}_{10} a_{10} \ell\right) c+x_{1} \ell\left(1-\hat{\beta}_{00}-\hat{\beta}_{10}\right)\right]\right. \\
& \left.-\frac{d \ell}{d p_{1}}\left[a_{10}-\hat{T} \hat{a}_{01} \frac{x_{1}}{w_{1} c}\left(1-\hat{\beta}_{00}-\hat{\beta}_{10}\right)\right]\right\}-\frac{y_{1}}{p_{1}}
\end{aligned}
$$


with $T=\left(a_{11} a_{00}-a_{10} a_{01}\right)^{-1}, \hat{T}=\left(\hat{a}_{11} \hat{a}_{00}-\hat{a}_{10} \hat{a}_{01}\right)^{-1}$ and

$$
\begin{aligned}
D & =1+\frac{T \hat{T} p_{1}}{w_{0} w_{1} c y_{1}}\left[\left(1-\hat{\beta}_{00}-\hat{\beta}_{10}\right)\left(\hat{\beta}_{01} a_{01} x_{1}+\hat{\beta}_{11} a_{11} \ell\right) y_{1}\right. \\
& +\left(1-\hat{\beta}_{01}-\hat{\beta}_{11}\right)\left(\hat{\beta}_{00} a_{00} x_{1}+\hat{\beta}_{10} a_{10} \ell\right) c \\
& \left.+x_{1} \ell\left(1-\hat{\beta}_{00}-\hat{\beta}_{10}\right)\left(1-\hat{\beta}_{01}-\hat{\beta}_{11}\right)\right]
\end{aligned}
$$

Substituting these expressions into (31) we get

$$
\begin{aligned}
\frac{d w_{0}}{d p_{1}} & =-\frac{\hat{T}}{D}\left\{\hat{a}_{10}-\frac{T}{w_{1} c}\left(1-\hat{\beta}_{00}-\hat{\beta}_{10}\right) a_{01} x_{1}\right. \\
& +\frac{d \ell}{d p_{1}} \frac{T p_{1}}{w_{1} c y_{1}}\left[\left(1-\hat{\beta}_{01}-\hat{\beta}_{11}\right) \hat{\beta}_{10} a_{10} c+\left(1-\hat{\beta}_{00}-\hat{\beta}_{10}\right) \hat{\beta}_{11} a_{11} y_{1}\right. \\
& \left.\left.+x_{1}\left(1-\hat{\beta}_{00}-\hat{\beta}_{10}\right)\left(1-\hat{\beta}_{01}-\hat{\beta}_{11}\right)\right]\right\} \\
\frac{d w_{1}}{d p_{1}} & =\frac{\hat{T}}{D}\left\{\hat{a}_{00}+\frac{T}{w_{0} c}\left(1-\hat{\beta}_{00}-\hat{\beta}_{10}\right) a_{11} \ell\right. \\
& \left.+\frac{d \ell}{d p_{1}} \frac{T p_{1}}{w_{0} c y_{1}}\left[\left(1-\hat{\beta}_{01}-\hat{\beta}_{11}\right) \hat{\beta}_{00} a_{10} c+\left(1-\hat{\beta}_{00}-\hat{\beta}_{10}\right) \hat{\beta}_{01} a_{11} y_{1}\right]\right\}
\end{aligned}
$$

Proceeding similarly, substituting expressions (33) and (34) into (4) and solving for the derivatives of the two ouput levels with respect to the stock of capital under the restriction $d p_{1}=0$, we now get:

$$
\begin{aligned}
& \frac{d c}{d x_{1}}=-T a_{01}\left\{1+\frac{x_{1}}{w_{1}} \frac{d w_{1}}{d x_{1}}-\frac{a_{11}}{a_{01}}\left(\frac{d \ell}{d x_{1}}+\frac{\ell}{w_{0}} \frac{d w_{0}}{d x_{1}}\right)\right\} \\
& \frac{d y_{1}}{d x_{1}}=T a_{00}\left\{1+\frac{x_{1}}{w_{1}} \frac{d w_{1}}{d x_{1}}-\frac{a_{10}}{a_{00}}\left(\frac{d \ell}{d x_{1}}+\frac{\ell}{w_{0}} \frac{d w_{0}}{d x_{1}}\right)\right\}
\end{aligned}
$$

Substituting these two expressions into (32) and solving for the derivatives of the wage rate and the rental rate with respect to the capital stock yields

$$
\begin{aligned}
\frac{d w_{0}}{d x_{1}} & =\frac{T \hat{T} p_{1}}{w_{1} c y_{1}}\left\{\left(1-\hat{\beta}_{00}-\hat{\beta}_{10}\right) \hat{\beta}_{11} a_{01} y_{1}+\left(1-\hat{\beta}_{01}-\hat{\beta}_{11}\right) \hat{\beta}_{10} a_{00} c\right. \\
& -\frac{d \ell}{d x_{1}}\left[\left(1-\hat{\beta}_{00}-\hat{\beta}_{10}\right) \hat{\beta}_{11} a_{11} y_{1}+\left(1-\hat{\beta}_{01}-\hat{\beta}_{11}\right) \hat{\beta}_{10} a_{10} c\right. \\
& \left.\left.+x_{1}\left(1-\hat{\beta}_{00}-\hat{\beta}_{10}\right)\left(1-\hat{\beta}_{01}-\hat{\beta}_{11}\right)\right]\right\} \\
\frac{d w_{1}}{d x_{1}} & =-\frac{T \hat{T} p_{1}}{w_{0} c y_{1}}\left\{\left(1-\hat{\beta}_{00}-\hat{\beta}_{10}\right) \hat{\beta}_{01} a_{01} y_{1}+\left(1-\hat{\beta}_{01}-\hat{\beta}_{11}\right) \hat{\beta}_{00} a_{00} c\right. \\
& +\ell\left(1-\hat{\beta}_{00}-\hat{\beta}_{10}\right)\left(1-\hat{\beta}_{01}-\hat{\beta}_{11}\right) \\
& \left.-\frac{d \ell}{d x_{1}}\left[\left(1-\hat{\beta}_{00}-\hat{\beta}_{10}\right) \hat{\beta}_{01} a_{11} y_{1}+\left(1-\hat{\beta}_{01}-\hat{\beta}_{11}\right) \hat{\beta}_{00} a_{10} c\right]\right\}
\end{aligned}
$$


Finally, substituting these two expressions back in (38), we obtain

$$
\begin{aligned}
\frac{d c}{d x_{1}} & =-\frac{T}{D}\left\{a_{01}-\frac{\hat{T} p_{1}}{w_{0} w_{1} y_{1}}\left(1-\hat{\beta}_{01}-\hat{\beta}_{11}\right) \hat{\beta}_{10} \ell\right. \\
& \left.-\frac{d \ell}{d x_{1}}\left[a_{11}+\frac{\hat{T} p_{1}}{w_{0} w_{1} y_{1}}\left(1-\hat{\beta}_{01}-\hat{\beta}_{11}\right) \hat{\beta}_{00} x_{1}\right]\right\} \\
\frac{d y_{1}}{d x_{1}} & =\frac{T}{D}\left\{a_{00}+\frac{\hat{T} p_{1}}{w_{0} w_{1} c}\left(1-\hat{\beta}_{00}-\hat{\beta}_{10}\right) \hat{\beta}_{11} \ell\right. \\
& \left.-\frac{d \ell}{d x_{1}}\left[a_{10}-\frac{\hat{T} p_{1}}{w_{0} w_{1} c}\left(1-\hat{\beta}_{00}-\hat{\beta}_{10}\right) \hat{\beta}_{01} x_{1}\right]\right\}
\end{aligned}
$$

Notice that if we assume constant social returns, i.e. $1-\hat{\beta}_{00}-\hat{\beta}_{10}=1-\hat{\beta}_{01}-$ $\hat{\beta}_{11}=0$, then these derivatives correspond to the Rybczynski effects either when labor is inelastic, i.e. $\gamma=+\infty$, or when the utility function is linear with respect to consumption, i.e. $\sigma=0$, as in these two cases $d \ell / d x_{1}=0$ (see (42) below).

We have finally to derive the expressions of $d \ell / d x_{1}$ and $d \ell / d p_{1}$. Total differenciation of equation (10) gives:

$$
\gamma d \ell=\frac{\ell}{w_{0}}\left(\frac{d w_{0}}{d x_{1}} d x_{1}+\frac{d w_{0}}{d p_{1}} d p_{1}\right)-\sigma \frac{\ell}{c}\left(\frac{d c}{d x_{1}} d x_{1}+\frac{d c}{d p_{1}} d p_{1}\right)
$$

Assume first that $d p_{1}=0$. We then get:

$$
\frac{d \ell}{d x_{1}}=\frac{\ell}{\gamma}\left(\frac{1}{w_{0}} \frac{d w_{0}}{d x_{1}}-\frac{\sigma}{c} \frac{d c}{d x_{1}}\right)
$$

Substituting $d w_{0} / d x_{1}$ from (39) and $d c / d x_{1}$ from (40) in (42) gives

$$
\begin{aligned}
\frac{d \ell}{d x_{1}} & =\frac{\ell}{\gamma} \frac{T}{D F}\left\{\sigma \frac{a_{01}}{c}+\frac{\hat{T} p_{1}}{w_{0} w_{1} c y_{1}}\left[\left(1-\hat{\beta}_{00}-\hat{\beta}_{10}\right) \hat{\beta}_{11} a_{01} y_{1}\right.\right. \\
& \left.\left.-\left(1-\hat{\beta}_{01}-\hat{\beta}_{11}\right) \hat{\beta}_{10}\left(\sigma \ell-a_{00} c\right)\right]\right\}
\end{aligned}
$$

with

$$
\begin{aligned}
F & =1+\frac{\ell T}{\gamma D}\left\{\sigma \frac{a_{11}}{c}+\frac{\hat{T} p_{1}}{w_{0} w_{1} c y_{1}}\left[\left(1-\hat{\beta}_{01}-\hat{\beta}_{11}\right)\left(\sigma \hat{\beta}_{00} x_{1}+\hat{\beta}_{10} a_{10} c\right)\right.\right. \\
& \left.\left.+\left(1-\hat{\beta}_{00}-\hat{\beta}_{10}\right) \hat{\beta}_{11} a_{11} y_{1}+x_{1}\left(1-\hat{\beta}_{00}-\hat{\beta}_{10}\right)\left(1-\hat{\beta}_{01}-\hat{\beta}_{11}\right)\right]\right\}
\end{aligned}
$$

Assume finally that $d x_{1}=0$. We then get

$$
\frac{d \ell}{d p_{1}}=\frac{\ell}{\gamma}\left(\frac{1}{w_{0}} \frac{d w_{0}}{d p_{1}}-\frac{\sigma}{c} \frac{d c}{d p_{1}}\right)
$$

Substituting $d w_{0} / d p_{1}$ from (37) and $d c / d p_{1}$ from (35) in (45) gives 


$$
\begin{aligned}
\frac{d \ell}{d p_{1}} & =\frac{\ell}{\gamma} \frac{T \hat{T}}{D F}\left\{\frac{1}{w_{0} w_{1} c}\left[\sigma\left(\hat{\beta}_{00} a_{01} x_{1}+\hat{\beta}_{10} a_{11} \ell\right)+\left(1-\hat{\beta}_{00}-\hat{\beta}_{10}\right) a_{01} x_{1}\right]\right. \\
& \left.\left.-\frac{\hat{a}_{10}}{T w_{0}}\right]\right\}
\end{aligned}
$$

The expressions of $\mathcal{D}(\sigma, \gamma)$ and $\mathcal{T}(\sigma, \gamma)$ can be obtained after substitution of (35), (37), (39), (40), (43) and (46) into (18).

\subsection{Proof of Theorem 1}

Let $\gamma=+\infty$. We then conclude from (43), (44) and (46) that $d \ell / d x_{1}=$ $d \ell / d p_{1}=0$ and $F=1$. We may also derive useful expressions for the wage rate and the rental rate for capital. Indeed, using the first order conditions (22), the expression of the stationary agregate capital stock (29) with $\ell^{*}=1$, (26) and (28) we get

$$
\begin{aligned}
& w_{0}=c \beta_{00} \frac{\delta+g\left(1-\beta_{11} b\right)}{\delta+g\left(1-\beta_{11}\right)} \\
& w_{1}=\frac{c}{x_{1}} \beta_{10} \frac{\delta+g}{\delta+g\left(1-\beta_{11}\right)}
\end{aligned}
$$

i) If $1-\hat{\beta}_{00}-\hat{\beta}_{10}=1-\hat{\beta}_{01}-\hat{\beta}_{11}=0$, then $D=1$ and $d \omega_{0} / d x_{1}=$ $d \omega_{1} / d x_{1}=0$. The proof of this result is provided in Garnier, Nishimura and Venditti [9]. Notice that the critical bound $\sigma^{*}$ is the value of $\sigma$ such that $E=0$, and is equal to

$$
\sigma^{*}=\frac{c^{*}}{p_{1}^{*}\left(\partial c / \partial p_{1}\right)}
$$

From (35), we get

$$
\frac{d c}{d p_{1}}=-\frac{T \hat{T}}{w_{0} w_{1}}\left(\hat{\beta}_{00} a_{01} x_{1}+\hat{\beta}_{10} a_{11} \ell\right)
$$

Using (47), we then derive

$$
\sigma^{*}=-\frac{\left(\beta_{00} \beta_{11}-\beta_{10} \beta_{01}\right)\left(\hat{\beta}_{00} \hat{\beta}_{11}-\hat{\beta}_{10} \hat{\beta}_{01}\right)\left[\delta+g\left(1-\beta_{11}\right)\right]}{\hat{\beta}_{00} \beta_{10} \beta_{01}(\delta+g)+\hat{\beta}_{10} \beta_{00} \beta_{11}\left[\delta+g\left(1-\beta_{11} b\right)\right]} \equiv \hat{\sigma}
$$

ii) Assume now that $1-\hat{\beta}_{00}-\hat{\beta}_{10}<0$ and $1-\hat{\beta}_{01}-\hat{\beta}_{11}=0$. As $b<0$ and $\hat{b}>0$ we get $T \hat{T}<0$ and thus $D>0$. Consider the partial derivative $d y_{1} / d x_{1}$ as given in (40). Using the definition of input coefficients at the social level (5) we get with $\ell^{*}=1$ :

From (27) we know that

$$
\frac{d y_{1}}{d x_{1}}=\frac{T}{D c}\left\{a_{00} c+\frac{\left(1-\hat{\beta}_{00}-\hat{\beta}_{10}\right) \hat{\beta}_{11}}{\hat{\beta}_{00} \hat{\beta}_{11}-\hat{\beta}_{10} \hat{\beta}_{01}}\right\}
$$

$$
\frac{a_{11} a_{00}}{a_{10} a_{01}}=\frac{\hat{\beta}_{00} \hat{\beta}_{11}}{\hat{\beta}_{10} \hat{\beta}_{01}}
$$


From Lemma 1 we also derive that at the steady state $a_{10} c=x_{1}\left(1-g a_{11}\right)$. But we know from (23), (25) and (29) that

$$
a_{11}=\frac{\beta_{11}}{\delta+g}, \quad \frac{a_{01}}{a_{11}}=\left(\frac{\beta_{11}}{\delta+g}\right)^{-\frac{1}{\hat{\beta}_{01}}}
$$

and

$$
x_{1}^{*}=\frac{\left(\frac{\beta_{10} \beta_{01}}{\beta_{00} \beta_{11}}\right)\left(\frac{\beta_{11}}{\delta+g}\right)^{\frac{1}{\hat{\beta}_{01}}}}{\left(1-\frac{\beta_{11}}{\delta+g} g b\right)}
$$

We then get from all this and Proposition 2

$$
\frac{d y_{1}}{d x_{1}}=\frac{T}{D c}\left\{\frac{\delta+g\left(1-\beta_{11}\right)}{\delta+g\left(1-\beta_{11} b\right)}+\frac{\left(1-\hat{\beta}_{00}-\hat{\beta}_{10}\right)}{\hat{b} \hat{\beta}_{00}}\right\}
$$

As $b<0$ we have $T<0$. It follows that $d y / d x_{1}<0$ if

$$
\hat{b}>-\frac{\left(1-\hat{\beta}_{00}-\hat{\beta}_{10}\right)\left[\delta+g\left(1-\beta_{11} b\right)\right]}{\hat{\beta}_{00}\left[\delta+g\left(1-\beta_{11}\right)\right]} \equiv \underline{\hat{b}}
$$

Consider the partial derivative $d w_{1} / d p_{1}$ as given in (37). We know that

$$
\hat{a}_{00}=\frac{\hat{\beta}_{00}}{\beta_{00}} a_{00}=\frac{\hat{\beta}_{00}}{\beta_{00}} \frac{a_{10} a_{01}}{a_{11}} \frac{\hat{\beta}_{00} \hat{\beta}_{11}}{\hat{\beta}_{10} \hat{\beta}_{01}}
$$

Proceeding as previously we then derive

$$
\frac{d w_{1}}{d p_{1}}=\frac{\hat{T}}{D c \beta_{00}}\left\{\hat{\beta}_{00} \frac{\delta+g\left(1-\beta_{11}\right)}{\delta+g\left(1-\beta_{11} b\right)}+\frac{\left(1-\hat{\beta}_{00}-\hat{\beta}_{10}\right)}{b}\right\}
$$

Using the steady state relationships (15) we get

$$
\delta+g-\frac{d w_{1}}{d p_{1}}=\frac{\delta+g}{D c}\left\{D c-\frac{w_{0}}{\left(\hat{\beta}_{00} \hat{\beta}_{11}-\hat{\beta}_{10} \hat{\beta}_{01}\right) \beta_{00}}\left[\hat{\beta}_{00} \frac{\delta+g\left(1-\beta_{11}\right)}{\delta+g\left(1-\beta_{11} b\right)}+\frac{\left(1-\hat{\beta}_{00}-\hat{\beta}_{10}\right)}{b}\right]\right\}
$$

It follows then from $(47)$

$\delta+g-\frac{d w_{1}}{d p_{1}}=\frac{\delta+g}{D}\left\{D-\frac{1}{\hat{\beta}_{00} \hat{\beta}_{11}-\hat{\beta}_{10} \hat{\beta}_{01}}\left[\hat{\beta}_{00}+\frac{\left(1-\hat{\beta}_{00}-\hat{\beta}_{10}\right)\left[\delta+g\left(1-\beta_{11} b\right)\right]}{b\left[\delta+g\left(1-\beta_{11}\right)\right]}\right]\right\}$

From (36) we get

$$
D=1+\frac{1-\hat{\beta}_{00}-\hat{\beta}_{10}}{\left(\beta_{00} \beta_{11}-\beta_{10} \beta_{01}\right)\left(\hat{\beta}_{00} \hat{\beta}_{11}-\hat{\beta}_{10} \hat{\beta}_{01}\right)}\left[\hat{\beta}_{01} \beta_{01} \frac{w_{1} x_{1}}{c}+\hat{\beta}_{11} \beta_{11} \frac{w_{0}}{c}\right]
$$

We finally derive from (47)

$$
D=1+\frac{\left(1-\hat{\beta}_{00}-\hat{\beta}_{10}\right)\left[\hat{\beta}_{01}(1-b)(\delta+g)+\hat{\beta}_{11}\left[\delta+g\left(1-\beta_{11} b\right)\right]\right]}{b\left(\hat{\beta}_{00} \hat{\beta}_{11}-\hat{\beta}_{10} \hat{\beta}_{01}\right)\left[\delta+g\left(1-\beta_{11}\right)\right]}
$$

Substituting this expression into (53) finally gives after simplifications:

$$
\delta+g-\frac{d w_{1}}{d p_{1}}=-\frac{(\delta+g) \hat{\beta}_{01}}{D\left(\hat{\beta}_{00} \hat{\beta}_{11}-\hat{\beta}_{10} \hat{\beta}_{01}\right)}<0
$$

as $\hat{b}<0$.

Consider the partial derivative $d w_{1} / d x_{1}$ as given in (39). We get 


$$
\frac{d w_{1}}{d x_{1}}=-\frac{T \hat{T} p_{1}}{w_{0} c y_{1}}\left(1-\hat{\beta}_{00}-\hat{\beta}_{10}\right) \hat{\beta}_{01} a_{01} y_{1}
$$

As $b<0, \hat{b}>0$ and thus $T \hat{T}<0$ we conclude that $d w_{1} / d x_{1}<0$.

Consider the partial derivative $d y_{1} / d p_{1}$ as given in (35). We get:

$$
\frac{d y_{1}}{d p_{1}}=\frac{(\delta+g)\left[\hat{\beta}_{00} \beta_{00}+\hat{\beta}_{10} \beta_{10} \frac{w_{0}}{w_{1} x_{1}}+\frac{w_{0}}{c}\left(1-\hat{\beta}_{00}-\hat{\beta}_{10}\right)\right]}{D\left(\beta_{00} \beta_{11}-\beta_{10} \beta_{01}\right)\left(\hat{\beta}_{00} \hat{\beta}_{11}-\hat{\beta}_{10} \hat{\beta}_{01}\right)} \frac{x_{1}}{p_{1}}-g \frac{x_{1}}{p_{1}}
$$

Using again (47), we obtain

$$
\begin{aligned}
\frac{d y_{1}}{d p_{1}} & =\frac{\beta_{00}\left[\hat{\beta}_{00}\left[\delta+g\left(1-\beta_{11}\right)\right](\delta+g)+\hat{\beta}_{10}\left[\delta+g\left(1-\beta_{11} b\right)\right]\left[\delta+g\left(1-\beta_{11}\right)\right]+\left(1-\hat{\beta}_{00}-\hat{\beta}_{10}\right)\left[\delta+g\left(1-\beta_{11} b\right)\right](\delta+g)\right]}{D\left(\beta_{00} \beta_{11}-\beta_{10} \beta_{01}\right)\left(\hat{\beta}_{00} \hat{\beta}_{11}-\hat{\beta}_{10} \hat{\beta}_{01}\right)\left[\delta+g\left(1-\beta_{11}\right)\right]} \frac{x_{1}}{p_{1}} \\
& -g \frac{x_{1}}{p_{1}} \\
& =\frac{\beta_{00}\left[-(\delta+g)(1-b) \hat{\beta}_{00} \beta_{11} g+\left[\delta+g\left(1-\beta_{11} b\right)\right] \hat{\beta}_{10} \beta_{11} g+\left[\delta+g\left(1-\beta_{11} b\right)\right](\delta+g)\right]}{D\left(\beta_{00} \beta_{11}-\beta_{10} \beta_{01}\right)\left(\hat{\beta}_{00} \hat{\beta}_{11}-\hat{\beta}_{10} \hat{\beta}_{01}\right)\left[\delta+g\left(1-\beta_{11}\right)\right]} \frac{x_{1}}{p_{1}}-g \frac{x_{1}}{p_{1}}
\end{aligned}
$$

Notice then that if $g=0$, we get

$$
\frac{d y_{1}}{d p_{1}}=\frac{\beta_{00} \delta}{D\left(\beta_{00} \beta_{11}-\beta_{10} \beta_{01}\right)\left(\hat{\beta}_{00} \hat{\beta}_{11}-\hat{\beta}_{10} \hat{\beta}_{01}\right)} \frac{x_{1}}{p_{1}}<0
$$

Therefore, there exists $\bar{g}>0$ such that when $g \in(0, \bar{g}), d y_{1} / d p_{1}<0$.

Let us then assume that $\sigma \in\left[0, \sigma^{*}\right)$, with $\sigma^{*}$ as defined by (48) so that $E>0$. Using (49), (50) and (54) we derive that

$$
\sigma^{*}=\hat{\sigma}-\frac{\left(1-\hat{\beta}_{00}-\hat{\beta}_{10}\right)\left[\hat{\beta}_{01}(1-b)(\delta+g)+\hat{\beta}_{11}\left[\delta+g\left(1-\beta_{11} b\right)\right]\right]}{\hat{\beta}_{00}(1-b)(\delta+g)+\hat{\beta}_{10}\left[\delta+g\left(1-\beta_{11} b\right)\right]}
$$

From all these results we conclude that if $b<0, \hat{b}>\underline{\hat{b}}$ with $\underline{\hat{b}}$ as defined by $(52), g \in(0, \bar{g})$ and $\sigma \in\left[0, \sigma^{*}\right)$, then $\mathcal{D}(\sigma,+\infty)>0$.

Notice now from (35) and (40) that

$$
\frac{d c}{d p_{1}}=-\frac{T}{D} \frac{\hat{T}}{w_{0} w_{1}}\left(\hat{\beta}_{00} a_{01} x_{1}+\hat{\beta}_{10} a_{11} \ell\right)>0, \quad \frac{d c}{d x_{1}}=-\frac{T}{D} a_{01}>0
$$

As $\sigma \in\left[0, \sigma^{*}\right)$ we derive from (18) that

$$
\mathcal{T}(\sigma,+\infty)<\mathcal{T}(\bar{\sigma},+\infty)=\frac{1}{E}\left\{\delta+g-\frac{\partial w_{1}}{\partial p_{1}}+\frac{1}{\partial c / \partial p_{1}} \frac{\partial c}{\partial x_{1}} \frac{\partial y_{1}}{\partial p_{1}}\right\}<0
$$

The result follows.

iii) Assume finally that $1-\hat{\beta}_{00}-\hat{\beta}_{10}=0$ and $1-\hat{\beta}_{01}-\hat{\beta}_{11}<0$. Again, as $b<0$ and $\hat{b}>0$ we get $T \hat{T}<0$ and thus $D>0$. Consider the partial derivatives as given in (35), (39) and (40). We easily get 


$$
\begin{aligned}
& \frac{d y_{1}}{d p_{1}}=\frac{T \hat{T}}{D w_{0} w_{1} c}\left(\hat{\beta}_{00} a_{00} x_{1}+\hat{\beta}_{10} a_{10} \ell\right) c-\frac{y_{1}}{p_{1}}<0 \\
& \frac{d c}{d p_{1}}=-\frac{T \hat{T}}{D w_{0} w_{1}}\left(\hat{\beta}_{00} a_{01} x_{1}+\hat{\beta}_{10} a_{11} \ell\right)>0 \\
& \frac{d w_{1}}{d x_{1}}=-\frac{T \hat{T} p_{1}}{w_{0} c}\left(1-\hat{\beta}_{00}-\hat{\beta}_{10}\right) \hat{\beta}_{01} a_{01}<0 \\
& \frac{d y_{1}}{d x_{1}}=\frac{T}{D} a_{00}<0 \\
& \frac{d c}{d x_{1}}=-\frac{T}{D}\left\{a_{01}-\frac{\hat{T} p_{1}}{w_{0} w_{1} y_{1}}\left(1-\hat{\beta}_{01}-\hat{\beta}_{11}\right) \hat{\beta}_{10}\right\}>0
\end{aligned}
$$

Consider now the partial derivative $d w_{1} / d p_{1}$ as given in (37). We derive at the steady state

$$
\delta+g-\frac{d w_{1}}{d p_{1}}=\frac{(\delta+g)}{D}\left\{D-\frac{\hat{\beta}_{00}}{\hat{\beta}_{00} \hat{\beta}_{11}-\hat{\beta}_{10} \hat{\beta}_{01}}\right\}
$$

From (36) we get

$$
D=1+\frac{\left(1-\hat{\beta}_{10}-\hat{\beta}_{11}\right)(\delta+g)}{\left(\hat{\beta}_{00} \hat{\beta}_{11}-\hat{\beta}_{10} \hat{\beta}_{01}\right)\left(\hat{\beta}_{00} \hat{\beta}_{11}-\hat{\beta}_{10} \hat{\beta}_{01}\right) g}\left[\hat{\beta}_{00} \beta_{00}+\hat{\beta}_{10} \beta_{10} \frac{w_{0}}{w_{1} x_{1}}\right]
$$

Using (47), we obtain after simplifications

$$
D=1+\frac{\left(1-\hat{\beta}_{10}-\hat{\beta}_{11}\right)}{b\left(\hat{\beta}_{00} \hat{\beta}_{11}-\hat{\beta}_{10} \hat{\beta}_{01}\right) \beta_{11} g}\left[\delta+g\left(1-\hat{\beta}_{10} \beta_{11} b\right)\right]
$$

We then derive

$$
\delta+g-\frac{d w_{1}}{d p_{1}}=\frac{(\delta+g)}{D\left(\hat{\beta}_{00} \hat{\beta}_{11}-\hat{\beta}_{10} \hat{\beta}_{01}\right)}\left\{\frac{\left(1-\hat{\beta}_{10}-\hat{\beta}_{11}\right)(\delta+g)-\left(1-\hat{\beta}_{11}\right) b \beta_{11} g}{b \beta_{11} g}\right\}
$$

As $b<0$, it follows that $\delta+g-d w_{1} / d p_{1}<$ if

$$
b<\frac{\left(1-\hat{\beta}_{10}-\hat{\beta}_{11}\right)(\delta+g)}{\left(1-\hat{\beta}_{11}\right) \beta_{11} g} \equiv \bar{b}
$$

Let us then assume that $\sigma \in\left[0, \sigma^{*}\right)$, with $\sigma^{*}$ as defined by (48) so that $E>0$. Using (49), (50) and (54) we derive that

$$
\sigma^{*}=\hat{\sigma}-\frac{\delta+g\left(1-\beta_{11}\right)}{g} \frac{\left(1-\hat{\beta}_{01}-\hat{\beta}_{11}\right)\left[\delta+g\left(1-\hat{\beta}_{10} \beta_{11} b\right)\right]}{\left[\hat{\beta}_{00}(1-b)(\delta+g)+\hat{\beta}_{10}\left[\delta+g\left(1-\beta_{11} b\right)\right]\right] \beta_{11}}
$$

From all these results we conclude that if $b<\bar{b}, \hat{b}>0$, and $\sigma \in\left[0, \sigma^{*}\right)$, then $\mathcal{D}(\sigma,+\infty)>0$. The result follows using again (56). 


\subsection{Proof of Theorem 2}

Let $\gamma=0$. We derive from (43) and (46) that

$$
\begin{aligned}
\frac{d \ell}{d x_{1}} & =\frac{1}{\tilde{F}}\left\{\sigma \frac{a_{01}}{c}+\frac{\hat{T} p_{1}}{w_{0} w_{1} c y_{1}}\left[\left(1-\hat{\beta}_{00}-\hat{\beta}_{10}\right) \hat{\beta}_{11} a_{01} y_{1}\right.\right. \\
& \left.\left.-\left(1-\hat{\beta}_{01}-\hat{\beta}_{11}\right) \hat{\beta}_{10}\left(\sigma \ell-a_{00} c\right)\right]\right\} \\
\frac{d \ell}{d p_{1}} & \left.=\frac{\hat{T}}{\widetilde{F}}\left\{\frac{1}{w_{0} w_{1} c}\left[\sigma\left(\hat{\beta}_{00} a_{01} x_{1}+\hat{\beta}_{10} a_{11} \ell\right)+\left(1-\hat{\beta}_{00}-\hat{\beta}_{10}\right) a_{01} x_{1}\right]-\frac{\hat{a}_{10}}{T w_{0}}\right]\right\}
\end{aligned}
$$

with

$$
\begin{aligned}
\tilde{F} & =\sigma \frac{a_{11}}{c}+\frac{\hat{T} p_{1}}{w_{0} w_{1} c y_{1}}\left[\left(1-\hat{\beta}_{01}-\hat{\beta}_{11}\right)\left(\sigma \hat{\beta}_{00} x_{1}+\hat{\beta}_{10} a_{10} c\right)\right. \\
& \left.+\left(1-\hat{\beta}_{00}-\hat{\beta}_{10}\right) \hat{\beta}_{11} a_{11} y_{1}+x_{1}\left(1-\hat{\beta}_{00}-\hat{\beta}_{10}\right)\left(1-\hat{\beta}_{01}-\hat{\beta}_{11}\right)\right]
\end{aligned}
$$

i) Assume that $1-\hat{\beta}_{01}-\hat{\beta}_{11}=0$. Consider first the case with $1-\hat{\beta}_{00}-$ $\hat{\beta}_{10}=0$. It follows that $D=1, d \omega_{0} / d x_{1}=d \omega_{1} / d x_{1}=0, \tilde{F}=\sigma a_{11} / c$ and thus

$$
\frac{d \ell}{d x_{1}}=\frac{a_{01}}{a_{11}}, \quad \frac{d \ell}{d p_{1}}=\frac{\hat{T}}{w_{0} a_{11}}\left[\frac{\hat{\beta}_{00} a_{01} x_{1}+\hat{\beta}_{10} a_{11} \ell}{w_{1}}-\frac{\hat{a}_{10} c}{T \sigma}\right]
$$

Therefore we derive from (35), (37) and (40)

$$
\frac{d c}{d p_{1}}-\frac{\hat{T} \hat{a}_{10} c}{\sigma w_{0}}, \quad \frac{d w_{1}}{d p_{1}}=\hat{T} \hat{a}_{00}, \quad \frac{d y_{1}}{d x_{1}}=\frac{1}{a_{11}}
$$

We also get from (14)

$$
E=1+\frac{\hat{T} p_{1} \hat{a}_{10}}{w_{0}}
$$

Using all these results and (18), we find after simplifications

$$
\mathcal{D}(\sigma, 0)=-\frac{\left(\frac{1-g a_{11}}{a_{11}}\right)\left[\hat{a}_{00}\left[1-(\delta+g) \hat{a}_{11}\right]+(\delta+g) \hat{a}_{10} \hat{a}_{01}\right]}{\hat{a}_{11} \hat{a}_{00}+\frac{\hat{a}_{10} \hat{a}_{11} w_{1}}{w_{0}}}<0
$$

so that indeterminacy is ruled out for any $\sigma \geq 0$.

Consider now the case with $1-\hat{\beta}_{00}-\hat{\beta}_{10}<0$. It is easy to show that $a_{01}-\left(d \ell / d x_{1}\right) a_{11}=0$. As a result we derive from (39) and (40), $d w_{1} / d x_{1}=0$ so that the determinant $\mathcal{D}(\sigma, 0)$ simplifies as

$$
\mathcal{D}(\sigma, 0)=\frac{1}{E}\left(\frac{\partial y_{1}}{\partial x_{1}}-g\right)\left(\delta+g-\frac{\partial w_{1}}{\partial p_{1}}\right)
$$

Moreover we get

$$
\tilde{F}=\frac{a_{11}}{c}\left[\sigma+\frac{\hat{T} p_{1}\left(1-\hat{\beta}_{00}-\hat{\beta}_{10}\right) \hat{\beta}_{11}}{w_{0} w_{1}}\right]
$$

Consider then the partial derivative $d y_{1} / d x_{1}$ as given in (40). We obtain

$$
\frac{d y_{1}}{d x_{1}}=\frac{1}{a_{11}} \text { and thus } \frac{d y_{1}}{d x_{1}}-g=\frac{1-g a_{11}}{a_{11}}>0
$$


Consider now the partial derivative $d w_{1} / d p_{1}$ as given in (37). We get after simplifications

and thus

$$
\frac{d w_{1}}{d p_{1}}=\frac{\hat{T}}{w_{0}} \frac{1-\hat{\beta}_{00}-\hat{\beta}_{10}+\sigma \hat{\beta}_{00}}{\frac{\hat{T} p_{1}\left(1-\hat{\beta}_{00}-\hat{\beta}_{10}\right) \hat{\beta}_{11}}{w_{0} w_{1}}+\sigma}
$$

$$
\delta+g-\frac{d w_{1}}{d p_{1}}=(\delta+g) \frac{\left(1-\hat{\beta}_{00}-\hat{\beta}_{10}\right)\left(\hat{\beta}_{11}-1\right)+\sigma\left[\hat{\beta}_{00} \hat{\beta}_{11}-\hat{\beta}_{10} \hat{\beta}_{01}-\hat{\beta}_{00}\right]}{\left(1-\hat{\beta}_{00}-\hat{\beta}_{10}\right) \hat{\beta}_{11}+\sigma\left(\hat{\beta}_{00} \hat{\beta}_{11}-\hat{\beta}_{10} \hat{\beta}_{01}\right)}
$$

Consider now the partial derivative $d c / d p_{1}$ as given in (35). We have:

$$
\frac{d c}{d p_{1}}=-\frac{\hat{T}}{w_{0}} \frac{\hat{a}_{10 c}}{\frac{\hat{T} p_{1}\left(1-\hat{\beta}_{00}-\hat{\beta}_{10}\right) \hat{\beta}_{11}}{w_{0} w_{1}}+\sigma}
$$

and thus

$$
E=1-\sigma \frac{p_{1}}{c} \frac{\partial c}{\partial p_{1}}=\frac{\left(1-\hat{\beta}_{00}-\hat{\beta}_{10}\right) \hat{\beta}_{11}+\sigma\left[\hat{\beta}_{00} \hat{\beta}_{11}-\hat{\beta}_{10} \hat{\beta}_{01}+\hat{\beta}_{10}\right]}{\left(1-\hat{\beta}_{00}-\hat{\beta}_{10}\right) \hat{\beta}_{11}+\sigma\left(\hat{\beta}_{00} \hat{\beta}_{11}-\hat{\beta}_{10} \hat{\beta}_{01}\right)}
$$

We may then derive that

$$
\mathcal{D}(\sigma, 0)=(\delta+g) \frac{1-g a_{11}}{a_{11}} \frac{\sigma\left[\hat{\beta}_{00} \hat{\beta}_{11}-\hat{\beta}_{10} \hat{\beta}_{01}-\hat{\beta}_{00}\right]-\left(1-\hat{\beta}_{00}-\hat{\beta}_{10}\right)\left(1-\hat{\beta}_{11}\right)}{\sigma\left[\hat{\beta}_{00} \hat{\beta}_{11}-\hat{\beta}_{10} \hat{\beta}_{01}+\hat{\beta}_{10}\right]+\left(1-\hat{\beta}_{00}-\hat{\beta}_{10}\right) \hat{\beta}_{11}}
$$

The numerator will be positive if and only if

$$
\sigma<\frac{\left(1-\hat{\beta}_{00}-\hat{\beta}_{10}\right)\left(1-\hat{\beta}_{11}\right)}{\hat{\beta}_{00} \hat{\beta}_{11}-\hat{\beta}_{10} \hat{\beta}_{01}-\hat{\beta}_{00}} \equiv \sigma_{N}
$$

while the denominator will be positive if and only if

$$
\sigma>\frac{\left(1-\hat{\beta}_{00}-\hat{\beta}_{10}\right) \hat{\beta}_{11}}{\hat{\beta}_{00} \hat{\beta}_{11}-\hat{\beta}_{10} \hat{\beta}_{01}+\hat{\beta}_{10}} \equiv \sigma_{D}
$$

But as $1-\hat{\beta}_{01}-\hat{\beta}_{11}=0$, we have $\sigma_{N}=\sigma_{D}$. It follows therefore that $\mathcal{D}(\sigma, 0)<0$ for any $\sigma \geq 0$.

ii) Assume now that $1-\hat{\beta}_{01}-\hat{\beta}_{11}<0$ and thus $1-\hat{\beta}_{00}-\hat{\beta}_{10}=0$. Consider the partial derivatives as given in (35), (37), (39) and (40). We easily get

$$
\begin{aligned}
& \frac{d y_{1}}{d p_{1}}=\frac{\hat{T}}{\tilde{F} w_{0} w_{1} c}\left(\hat{\beta}_{10} a_{10} c+\sigma \hat{\beta}_{00} x_{1}\right)-\frac{y_{1}}{p_{1}}, \frac{d c}{d p_{1}}=-\frac{\hat{T}}{\tilde{F} w_{0}} \hat{a}_{10} a_{11}, \frac{d w_{1}}{d p_{1}}=\sigma \frac{\hat{T}}{\tilde{F} c} \hat{a}_{00} a_{11} \\
& \frac{d w_{1}}{d x_{1}}=-\sigma \frac{\hat{T} p_{1}}{\tilde{F} w_{0} c y_{1}}\left(1-\hat{\beta}_{01}-\hat{\beta}_{11}\right) \hat{\beta}_{00}, \frac{d y_{1}}{d x_{1}}=\frac{\sigma}{\tilde{F} c}, \frac{d c}{d x_{1}}=-\frac{\hat{T} p_{1}}{\tilde{F} w_{0} w_{1} y_{1}}\left(1-\hat{\beta}_{01}-\hat{\beta}_{11}\right) \hat{\beta}_{10} \\
& \text { with } \\
& \qquad \tilde{F}=\frac{\sigma\left[\left(\hat{\beta}_{00} \hat{\beta}_{11}-\hat{\beta}_{10} \hat{\beta}_{01}\right) \beta_{11} g+(\delta+g)\left(1-\hat{\beta}_{01}-\hat{\beta}_{11}\right) \hat{\beta}_{00}\right]+\left[\delta+g\left(1-\beta_{11}\right)\right]\left(1-\hat{\beta}_{01}-\hat{\beta}_{11}\right) \hat{\beta}_{10}}{\left(\hat{\beta}_{00} \hat{\beta}_{11}-\hat{\beta}_{10} \hat{\beta}_{01}\right) g(\delta+g) c}
\end{aligned}
$$

From all these results we can compute 


$$
\begin{aligned}
E & =\frac{\sigma\left[\left(\hat{\beta}_{00} \hat{\beta}_{11}-\hat{\beta}_{10} \hat{\beta}_{01}+\hat{\beta}_{10}\right) \beta_{11} g+(\delta+g)\left(1-\hat{\beta}_{01}-\hat{\beta}_{11}\right) \hat{\beta}_{00}\right]+\left[\delta+g\left(1-\beta_{11}\right)\right]\left(1-\hat{\beta}_{01}-\hat{\beta}_{11}\right) \hat{\beta}_{10}}{\sigma\left[\left(\hat{\beta}_{00} \hat{\beta}_{11}-\hat{\beta}_{10} \hat{\beta}_{01}\right) \beta_{11} g+(\delta+g)\left(1-\hat{\beta}_{01}-\hat{\beta}_{11}\right) \hat{\beta}_{00}\right]+\left[\delta+g\left(1-\beta_{11}\right)\right]\left(1-\hat{\beta}_{01}-\hat{\beta}_{11}\right) \hat{\beta}_{10}} \\
& \equiv \frac{\mathcal{M}}{\mathcal{N}} \\
\frac{d y_{1}}{d x_{1}}-g & =g \frac{\sigma\left[\left(\hat{\beta}_{00} \hat{\beta}_{11}-\hat{\beta}_{10} \hat{\beta}_{01}\right)\left[\delta+g\left(1-\beta_{11}\right)\right]-(\delta+g)\left(1-\hat{\beta}_{01}-\hat{\beta}_{11}\right) \hat{\beta}_{00}\right]-\left[\delta+g\left(1-\beta_{11}\right)\right]\left(1-\hat{\beta}_{01}-\hat{\beta}_{11}\right) \hat{\beta}_{10}}{\mathcal{N}} \\
& \equiv g \frac{\mathcal{M} y_{1} / x_{1}}{\mathcal{N}} \\
\delta+g-\frac{d w_{1}}{d p_{1}} & =(\delta+g) \frac{\sigma\left[\left(\hat{\beta}_{00} \hat{\beta}_{11}-\hat{\beta}_{10} \hat{\beta}_{01}-\hat{\beta}_{00}\right) \beta_{11} g+(\delta+g)\left(1-\hat{\beta}_{01}-\hat{\beta}_{11}\right) \hat{\beta}_{00}\right]+\left[\delta+g\left(1-\beta_{11}\right)\right]\left(1-\hat{\beta}_{01}-\hat{\beta}_{11}\right) \hat{\beta}_{10}}{\mathcal{N}} \\
& \equiv(\delta+g) \frac{\mathcal{M}_{w_{1} / p_{1}}}{\mathcal{N}} \\
\frac{d y_{1}}{d p_{1}} & =x_{1} g \frac{\left[\delta+g\left(1-\beta_{11}\right)\right]\left(\hat{\beta}_{01}+\hat{\beta}_{11}\right) \hat{\beta}_{10}-\sigma\left[\left(\hat{\beta}_{00} \hat{\beta}_{11}-\hat{\beta}_{10} \hat{\beta}_{01}\right) \beta_{11} g-(\delta+g)\left(\hat{\beta}_{01}+\hat{\beta}_{11}\right) \hat{\beta}_{00}\right]}{p_{1} \mathcal{N}} \\
& \equiv x_{1} g \frac{\mathcal{M} y_{1} / p_{1}}{p_{1} \mathcal{N}} \\
\frac{d w_{1}}{d x_{1}} & =-\sigma \frac{w_{1}}{g x_{1}} \frac{\left(1-\hat{\beta}_{01}-\hat{\beta}_{11}\right) \hat{\beta}_{00} g(\delta+g)}{\mathcal{N}} \\
\frac{p_{1}}{c} \frac{d c}{d x_{1}} & =-\frac{p_{1}}{g x_{1}} \frac{\left(1-\hat{\beta}_{01}-\hat{\beta}_{11}\right) \hat{\beta}_{10} g(\delta+g)}{\mathcal{N}} \\
\frac{p_{1}}{c} \frac{d c}{d p_{1}} & =-\frac{\hat{\beta}_{10} \beta_{11} g}{\mathcal{N}}
\end{aligned}
$$

Notice that $\mathcal{M}>\mathcal{N}$, and as $\hat{b}>0, \mathcal{M}_{y_{1} / x_{1}}>0$ while $\mathcal{M}_{w_{1} / p_{1}}<0$. Moreover, we derive that $\mathcal{M}>0$ if

$$
\hat{b}>-\frac{(\delta+g)\left(1-\hat{\beta}_{01}-\hat{\beta}_{11}\right)}{\beta_{11} \hat{\beta}_{11} g}-\frac{\hat{\beta}_{10}}{\hat{\beta}_{00} \hat{\beta}_{11}} \equiv \hat{b}_{1}
$$

and

$$
\sigma>-\frac{\left[\delta+g\left(1-\beta_{11}\right)\right]\left(1-\hat{\beta}_{01}-\hat{\beta}_{11}\right) \hat{\beta}_{10}}{\left(\hat{\beta}_{00} \hat{\beta}_{11}-\hat{\beta}_{10} \hat{\beta}_{01}+\hat{\beta}_{10}\right) \beta_{11} g+(\delta+g)\left(1-\hat{\beta}_{01}-\hat{\beta}_{11}\right) \hat{\beta}_{00}} \equiv \sigma_{1}
$$

while $\mathcal{N}<0$ if

$$
\hat{b}>-\frac{(\delta+g)\left(1-\hat{\beta}_{01}-\hat{\beta}_{11}\right)}{\beta_{11} \hat{\beta}_{11} g} \equiv \hat{b}_{2}
$$

and

$$
\sigma<-\frac{\left[\delta+g\left(1-\beta_{11}\right)\right]\left(1-\hat{\beta}_{01}-\hat{\beta}_{11}\right) \hat{\beta}_{10}}{\left(\hat{\beta}_{00} \hat{\beta}_{11}-\hat{\beta}_{10} \hat{\beta}_{01}\right) \beta_{11} g+(\delta+g)\left(1-\hat{\beta}_{01}-\hat{\beta}_{11}\right) \hat{\beta}_{00}} \equiv \sigma_{2}
$$

Obviously we have $\hat{b}_{2}>\hat{b}_{1}$ and we can easily show that $\sigma_{2}>\sigma_{1}$. Notice also that when $\hat{b}>\hat{b}_{2}$ and $\sigma<\sigma_{2}$ then $\mathcal{M}_{y_{1} / p_{1}}>0$.

We may now compute the determinant:

$$
\mathcal{D}(\sigma, 0)=\frac{g(\delta+g)}{\mathcal{M N}}\left[\mathcal{M}_{y_{1} / x_{1}} \mathcal{M}_{w_{1} / p_{1}}-\sigma(\delta+g) \mathcal{M}_{y_{1} / p_{1}}\left(1-\hat{\beta}_{01}-\hat{\beta}_{11}\right) \hat{\beta}_{00}\right]
$$

Therefore, there exists $\epsilon_{1}>0$ such that $\mathcal{D}(\sigma, 0)>0$ if $\hat{\beta}_{01}+\hat{\beta}_{11} \in\left(1,1+\epsilon_{1}\right)$, $\hat{b}>\hat{b}_{2}$ and $\sigma \in\left(\sigma_{1}, \sigma_{2}\right)$.

Let us now compute the trace. We start with the following: 


$$
\begin{aligned}
\frac{\left[\left(\frac{\partial y_{1}}{\partial x_{1}}-g\right)+\left(\delta+g-\frac{\partial w_{1}}{\partial p_{1}}\right)\right]}{E} & =\frac{g \mathcal{M}_{y_{1} / x_{1}}+(\delta+g) \mathcal{M}_{w_{1} / p_{1}}}{\mathcal{M}} \\
& =\frac{\sigma\left[\left(\hat{\beta}_{00} \hat{\beta}_{11}-\hat{\beta}_{10} \hat{\beta}_{01}\right)\left[\delta\left(1+\hat{\beta}_{11}\right)+g\right] g+\delta(\delta+g)\left(1-\hat{\beta}_{01}-\hat{\beta}_{11}\right) \hat{\beta}_{00}\right]+\delta\left[\delta+g\left(1-\beta_{11}\right)\right]\left(1-\hat{\beta}_{01}-\hat{\beta}_{11}\right) \hat{\beta}_{10}}{\mathcal{M}} \\
& \equiv \mathcal{T}_{1}
\end{aligned}
$$

This expression is then negative if

$$
\hat{b}>-\frac{\delta(\delta+g)\left(1-\hat{\beta}_{01}-\hat{\beta}_{11}\right)}{\left[\delta\left(1+\hat{\beta}_{11}\right)+g\right] \hat{\beta}_{11} g} \equiv \hat{b}_{3}
$$

and

$$
\sigma<-\frac{\delta\left[\delta+g\left(1-\beta_{11}\right)\right]\left(1-\hat{\beta}_{01}-\hat{\beta}_{11}\right) \hat{\beta}_{10}}{\left(\hat{\beta}_{00} \hat{\beta}_{11}-\hat{\beta}_{10} \hat{\beta}_{01}\right)\left[\delta\left(1+\hat{\beta}_{11}\right)+g\right] g+\delta(\delta+g)\left(1-\hat{\beta}_{01}-\hat{\beta}_{11}\right) \hat{\beta}_{00}} \equiv \sigma_{3}
$$

with $\hat{b}_{2}>\hat{b}_{3}$ and $\sigma_{3}<\sigma_{2}$. Notice also that $\sigma_{1}<\sigma_{3}$ if

$$
\hat{b}<\frac{\delta \hat{\beta}_{10} \hat{\beta}_{11}}{(\delta+g) \hat{\beta}_{00} \hat{\beta}_{11}} \equiv \hat{b}_{4}
$$

It follows that there exists $\epsilon_{2}>0$ such that $\hat{b}_{4}>\hat{b}_{2}$ when $\hat{\beta}_{01}+\hat{\beta}_{11} \epsilon$ $\left(1,1+\epsilon_{2}\right)$. Consider then the second part of the trace:

$\frac{p_{1}^{*}}{E c^{*}}\left[\frac{\partial c}{\partial x_{1}} \frac{\partial y_{1}}{\partial p_{1}}-\frac{\partial c}{\partial p_{1}}\left(\frac{\partial y_{1}}{\partial x_{1}}-g\right)\right]=\frac{\hat{\beta}_{10} g\left[\beta_{11} g \mathcal{M}_{y_{1} / x_{1}}-(\delta+g)\left(1-\hat{\beta}_{01}-\hat{\beta}_{11}\right) \mathcal{M}_{y_{1} / p_{1}}\right]}{\mathcal{M N}} \equiv \mathcal{T}_{2}$ It follows that $\mathcal{T}_{2}<0$ if $\hat{b}>\hat{b}_{2}$ and $\sigma \in\left(\sigma_{1}, \sigma_{2}\right)$. By definition we have $\mathcal{T}(\sigma, 0)=\mathcal{T}_{1}+\sigma \mathcal{T}_{2}$. Notice that as $\sigma_{3}<\sigma_{2}$, if $\sigma=\sigma_{3}$, then $\mathcal{T}_{1}=0$ but $\mathcal{T}(\sigma, 0)=\mathcal{T}_{2}<0$. On the contrary, if $\sigma=\sigma_{2}$, then $\mathcal{N}=0$ and $\mathcal{T}_{2}=-\infty$ so that $\mathcal{T}(\sigma, 0)=-\infty$. Therefore, there exists $\tilde{\sigma} \in\left(\sigma_{3}, \sigma_{2}\right]$ such that when $\hat{b} \in\left(\hat{b}_{2}, \hat{b}_{4}\right), \mathcal{T}(\sigma, 0)<0$ for any $\sigma<\tilde{\sigma}$.

To conclude, let $\epsilon=\min \left\{\epsilon_{1}, \epsilon_{2}\right\}, \underline{\sigma}=\sigma_{1}$ and $\bar{\sigma}=\tilde{\sigma}$. Therefore, the steady state is locally indeterminate if $\hat{\beta}_{01}+\hat{\beta}_{11} \in(1,1+\epsilon), \hat{b} \in\left(\hat{b}_{2}, \hat{b}_{4}\right)$ and $\sigma \in(\underline{\sigma}, \bar{\sigma})$.

\subsection{Proof of Proposition 3}

Consider the expression $E=\mathcal{M} / \mathcal{N}$ given in the proof of Theorem 2. Assume that $E>0$. As $\mathcal{M}>\mathcal{N}$, this is obtained if either $\mathcal{N}>0$ or $\mathcal{M}<0$.

i) Assume first that $\mathcal{N}>0$, i.e. $\hat{b}>\hat{b}_{2}$ and $\sigma>\sigma_{2}$. As $\hat{b}>0$, we get $\mathcal{M}_{y_{1} / x_{1}}>0$ while $\mathcal{M}_{w_{1} / p_{1}}<0$. It follows from (60) that there exists $\epsilon>0$ such that if $\hat{\beta}_{01}+\hat{\beta}_{11} \in(1,1+\epsilon), \mathcal{D}(\sigma, 0)<0$.

ii) Asume now that $\mathcal{M}<0$, i.e. either $\hat{b}>\hat{b}_{1}$ and $\sigma<\sigma_{1}$, or $\hat{b} \in\left(0, \hat{b}_{1}\right)$. Again, as $\hat{b}>0$, we get $\mathcal{M}_{y_{1} / x_{1}}>0$ while $\mathcal{M}_{w_{1} / p_{1}}<0$. It follows from (60) that there exists $\epsilon>0$ such that if $\hat{\beta}_{01}+\hat{\beta}_{11} \in(1,1+\epsilon), \mathcal{D}(\sigma, 0)<0$. 


\subsection{Proof of Theorem 3}

Using Theorems 1 and 2, we may prove the different subcases of Theorem $3:$

i) Let $1-\hat{\beta}_{00}-\hat{\beta}_{10}=1-\hat{\beta}_{01}-\hat{\beta}_{11}=0$. The proof of this result is provided in Garnier, Nishimura and Venditti [9]. Notice that the bound $\sigma^{*}$ is given by (50).

ii) Assume now that $1-\hat{\beta}_{00}-\hat{\beta}_{10}<0$ and $1-\hat{\beta}_{01}-\hat{\beta}_{11}=0$. As shown in Theorem 1ii), when $\gamma=+\infty$, the steady state is locally indeterminate if

$$
b<0, \quad \hat{b}>-\frac{\left(1-\hat{\beta}_{00}-\hat{\beta}_{10}\right)\left[\delta+g\left(1-\beta_{11} b\right)\right]}{\hat{\beta}_{00}\left[\delta+g\left(1-\beta_{11}\right)\right]}, \quad g \in(0, \bar{g}) \text { and } \sigma \in\left[0, \sigma^{*}\right)
$$

where the bound $\sigma^{*}$ is given by (55). On the contrary, we have proved with Theorem 2ii) that when $\gamma=0$, the steady state is saddle-point stable for any $\sigma \geq 0$. The result follows.

iii) Assume finally that $1-\hat{\beta}_{00}-\hat{\beta}_{10}=0$ and $1-\hat{\beta}_{01}-\hat{\beta}_{11}<0$. As shown in Theorem 1iii), when $\gamma=+\infty$ the steady state is locally indeterminate if

$$
b<\frac{\left(1-\hat{\beta}_{01}-\hat{\beta}_{11}\right)(\delta+g)}{\left(1-\hat{\beta}_{11}\right) g}, \quad \hat{b}>0 \text { and } \sigma \in\left[0, \sigma^{*}\right)
$$

where the bound $\sigma^{*}$ is given by (57). Moreover, as shown in Theorem 2iii), when $\gamma=0$ the steady state is locally indeterminate if

$$
\hat{\beta}_{00}+\hat{\beta}_{10} \in(1,1+\epsilon), \quad \hat{b} \in\left(-\frac{\left(1-\hat{\beta}_{01}-\hat{\beta}_{11}\right)(\delta+g)}{\hat{\beta}_{11} \beta_{11} g}, \frac{\hat{\beta}_{01} \beta_{11} \delta}{\hat{\beta}_{00} \hat{\beta}_{11}(\delta+g)}\right) \text { and } \sigma \in(\underline{\sigma}, \bar{\sigma})
$$

with $\epsilon=\min \left\{\epsilon_{1}, \epsilon_{2}\right\}, \underline{\sigma}=\sigma_{1}$ and $\bar{\sigma}=\tilde{\sigma} \in\left(\sigma_{3}, \sigma_{2}\right]$ as defined in Section 5.4.

We easily derive that there exists $\epsilon_{3}>0$ such that $\sigma_{2}<\sigma^{*}$ if $\hat{\beta}_{01}+\hat{\beta}_{11} \epsilon$ $\left(1,1+\epsilon_{3}\right)$. As a result $\bar{\sigma}<\sigma^{*}$, and denoting now $\epsilon=\min \left\{\epsilon_{1}, \epsilon_{2}, \epsilon_{3}\right\}$, it follows that when $\hat{\beta}_{01}+\hat{\beta}_{11} \in(1,1+\epsilon)$, we may assume simultaneously all the following restrictions

$$
b<\frac{\left(1-\hat{\beta}_{01}-\hat{\beta}_{11}\right)(\delta+g)}{\left(1-\hat{\beta}_{11}\right) g}, \quad \hat{b} \in\left(-\frac{\left(1-\hat{\beta}_{01}-\hat{\beta}_{11}\right)(\delta+g)}{\hat{\beta}_{11} \beta_{11} g}, \frac{\hat{\beta}_{01} \beta_{11} \delta}{\hat{\beta}_{00} \hat{\beta}_{11}(\delta+g)}\right)
$$

and $\sigma \in(\underline{\sigma}, \bar{\sigma})$. The question is now to know whether local indeterminacy may occur for any $\gamma \geq 0$ when the above conditions are satisfied. The answer is negative. Indeed, when $\gamma=+\infty$, if $\sigma<\bar{\sigma}$, then as shown in Section $5.3, E\left(x_{1}^{*}, p_{1}^{*}\right)>0$. On the contrary, when $\gamma=0$, if $\hat{b}$ satisfies (61) and $\sigma \in(\underline{\sigma}, \bar{\sigma})$, then as shown in Section 5.4, $E\left(x_{1}^{*}, p_{1}^{*}\right)<0$. Notice finally that when $\sigma \in(\underline{\sigma}, \bar{\sigma}), E\left(x_{1}^{*}, p_{1}^{*}\right)$ is a continous function of $\gamma$. It follows that there exist intermediary values for $\gamma$ such that the determinant $\mathcal{D}(\sigma, \gamma)$ is negative. The result follows. 


\section{References}

[1] Basu, S., and J. Fernald (1997): "Returns to Scale in US Production: Estimates and Implications," Journal of Political Economy, 105, 249283.

[2] Benhabib, J., and R. Farmer (1994): "Indeterminacy and Increasing Returns," Journal of Economic Theory, 63, 19-41.

[3] Benhabib, J., and R. Farmer (1996): "Indeterminacy and Sector Specific Externalities," Journal of Monetary Economics, 37, 397-419.

[4] Benhabib, J. and R. Farmer (1999): "Indeterminacy and Sunspots in Macroeconomics," in Handbook of Macroeconomics, J.B. Taylor and M. Woodford (eds.), North-Holland, Amsterdam, 387-448.

[5] Benhabib, J., and K. Nishimura (1998): "Indeterminacy and Sunspots with Constant Returns," Journal of Economic Theory, 81, 58-96.

[6] Benhabib, J., K. Nishimura and A. Venditti (2002): "Indeterminacy and Cycles in Two-Sector Discrete-Time Model," Economic Theory, 20, 217-235.

[7] Blundell, R., and T. McCurdy (1999): "Labour Supply: a Review of Alternative Approaches." In O.Ashenfelter and D. Card (eds.), Handbook of Labor Economics, North-Holland, 1559-1695

[8] Garnier, J.P., K. Nishimura and A. Venditti (2007): "Capital-Labor Substitution and Indeterminacy in Continuous-Time Two-Sector Models," Advances in Mathematical Economics, 10, 31-49.

[9] Garnier, J.P., K. Nishimura and A. Venditti (2007): "Intertemporal Substitution in Consumption, Labor Supply Elasticity and Sunspot Fluctuations in Continuous-Time Models," International Journal of Economic Theory, 3, 235-259. 
[10] Grandmont, J.-M., P. Pintus and R. De Vilder (1998): "Capital-Labor Substitution and Competitive Nonlinear Endogenous Business Cycles," Journal of Economic Theory, 80, 14-59.

[11] Harrison, S. (2001): "Indeterminacy in a Model with Sector Specific Externalities," Journal of Economic Dynamics and Control, 25, 747764.

[12] Harrison, S., and M. Weder (2002): "Tracing Externalities as Sources of Indeterminacy," Journal of Economic Dynamics and Control, 26, 851-867.

[13] Lloyd-Braga, T., C. Nourry and A. Venditti (2006): "Indeterminacy with Small Externalities: the Role of Non-Separable Preferences," International Journal of Economic Theory, 2, 217-239.

[14] Nishimura, K., C. Nourry and A. Venditti (2007): "Indeterminacy in Aggregate Models with Small Externalities: an Interplay Between Preferences and Technology," GREQAM-KIER mimeo.

[15] Nishimura, K., and A. Venditti (2004): "Indeterminacy and the Role of Factor Substitutability," Macroeconomic Dynamics, 8, 436-465.

[16] Nishimura, K., and A. Venditti (2007): "Indeterminacy in DiscreteTime Infinite-Horizon Models with Non-Linear Utility and Endogenous Labor," Journal of Mathematical Economics, 43, 446-476.

[17] Pintus, P (2006): "Indeterminacy with Almost Constant Returns to Scale: Capital-Labor Substitution Matters", Economic Theory, 28, 633649.

[18] Wen, Y. (1998): "Capacity Utilization under Increasing Returns to Scale", Journal of Economic Theory, 81, 7-36. 